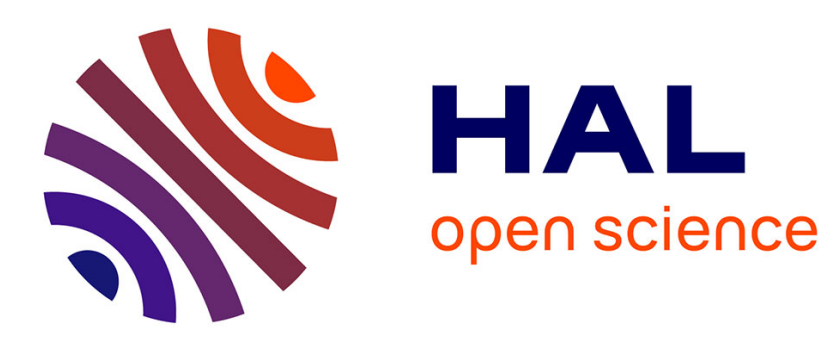

\title{
A multiscale mixed finite element method applied to the simulation of two-phase flows
}

Omar Duran, Philippe R B Devloo, Sonia M. Gomes, José B. Villegas

\section{To cite this version:}

Omar Duran, Philippe R B Devloo, Sonia M. Gomes, José B. Villegas. A multiscale mixed finite element method applied to the simulation of two-phase flows. 2021. hal-02464476v4

\section{HAL Id: hal-02464476 \\ https://hal.science/hal-02464476v4}

Preprint submitted on 21 May 2021

HAL is a multi-disciplinary open access archive for the deposit and dissemination of scientific research documents, whether they are published or not. The documents may come from teaching and research institutions in France or abroad, or from public or private research centers.
L'archive ouverte pluridisciplinaire HAL, est destinée au dépôt et à la diffusion de documents scientifiques de niveau recherche, publiés ou non, émanant des établissements d'enseignement et de recherche français ou étrangers, des laboratoires publics ou privés. 


\title{
A multiscale mixed finite element method applied to the simulation of two-phase flows
}

\author{
Omar Duran* $\quad$ Philippe R. B. Devloo ${ }^{\dagger} \quad$ Sônia M. Gomes ${ }^{\ddagger} \quad$ Jose Villegas $^{\S}$
}

May 20, 2021

\begin{abstract}
The multiscale hybrid mixed finite element method (MHM-H(div)), previously developed for Darcy's problems, is extended for coupled flow/pressure and transport system of two-phase flow equations on heterogeneous media under the effect of gravitational segregation. It is combined with an implicit transport solver in a sequential fully implicit (SFI) manner. The MHM-H(div) method is designed to cope with the complex geometry and inherent multiscale nature of the phenomena. The discretizations are based on a general domain partition formed by polyhedral subregions, where a hierarchy of meshes and approximation spaces are considered. The multiscale approach is applied to the flux/pressure kernel making use of coarse scale normal fluxes between subregions (trace variable). The fine-scale features inside each subregion are determined by resolving completely independent local Neumann problems, the boundary conditions being set by the trace variable, by the mixed finite element method using fine flux and pressure representations. These properties imply that the MHM-H(div) can be interpreted as a classical mixed formulation of the model problem in the whole domain, based on a $H$ (div)conforming space with normal components over the macro-partition interfaces constrained by the trace space, and showing divergence compatibility with the pressure space. Consequently, local mass conservation is observed at the micro-scale elements inside the subregions, an essential property for flows in heterogeneous media, and divergence-free constraint strongly enforced for incompressible flows. The efficient use of static condensation leads to a global system to be solved only in terms of primary degrees of freedom associated with the trace variable and of a piecewise constant pressure for each subregion. This procedure allows a substantial reduction of the dominant computational costs associated with the flux/pressure kernel embedded in the numerical model. An iterative coupling technique is adopted to solve the two-phase flow equations using a shared integration point memory implementation model. At each SFI time step, the efficiency iterative method for the transport equations is improved using a Quasi-Newton method with a simple but effective nonlinear acceleration. The numerical examples show that the proposed scheme is able to solve challenging coupled flow and transport problems.
\end{abstract}

Keywords : Multiscale hybrid method, Mixed finite elements, Two phase flow, Gravitational segregation, Sequential fully implicit strategy.

\section{Dedicated to Mary Wheeler on the occasion of her 80th birthday anniversary}

Prof. Wheeler is the seed of our research in mixed methods applied to the area of reservoir simulation. The authors and members of LabMeC are grateful for her groundbreaking research in this area.

\footnotetext{
*Centre d'Enseignement et de Recherche en Mathématiques et Calcul scientifique, CERMICS (ENPC), Université Paris-Est, 77455, Paris, France, E-mail address: omar.duran@enpc.fr

${ }^{\dagger}$ LabMeC-FEC-Universidade Estadual de Campinas, Campinas-SP, Brazil, E-mail address: phil@unicamp.br

¥IMECC-Universidade Estadual de Campinas, Campinas, SP, Brazil, E-mail address: soniag@unicamp.br

${ }^{\S}$ DEP-FEM-Universidade Estadual de Campinas, Campinas, SP, Brazil, E-mail address: j153310@unicamp.br
} 


\section{Introduction}

The extraction of petroleum is a process that involves different stages: exploration, development, production and abandonment of wells, as discussed in [1, 2, 3]. This sequence of processes occur in a dynamic form, where the information collected during each stage is assimilated, using a computational infrastructure with the objective of predicting the behavior and planning the development of an oil field. Thus, reservoir simulation is a tool that provides insights of this dynamic process during any stage of the oil extraction.

Several commercial software applied to reservoir engineering use technologies developed more than 50 years ago to approximate multiphase flow in heterogeneous porous media. Current techniques used by most industries are based on low-order numerical schemes (usually of finite differences or volumes). Thus, in order to obtain accurate solutions, typical simulation models are constructed with millions of cells. Additionally, many efforts in numerical simulation applied to petroleum engineering, such as history matching, optimization and uncertainty evaluation, require to perform a considerable number of simulations.

For such computational demand, the robustness and efficiency of a simulator is a crucial factor. Accurate approximations require larger computer resources than lower order models. Availability of such computer resources becomes crucial, specially if the model contains relevant solution features of different scales or different geometric dimensions. Moreover, many numerical simulators are unfit to include dimensional coupling (e.g. coupling 1D well flow, 2D fractures flow and a 3D reservoir flow). Trying to model problems with different scales with a non-adapted code leads to high resolution in the discretization, i.e. $10^{7}-10^{8}$ elements or simulation blocks [4]. In the presence of such large meshes, convergence becomes more difficult, rendering the simulation unfeasible.

In order to turn finer scale problems solvable, one can apply degrees-of-freedom reduction, either by grid upscaling or homogenization processes. These procedures rely on computing material properties or functions on coarse domain partitions [5, 6, 7]. However, such procedures are not robust nor flexible for reservoir simulation, presenting discrepancies with high permeability contrasts [7], requiring further studies to become a mature flow simulation technique.

An alternative is to include finer geocellular scales directly changing the computational model, leading to numerical schemes capable of incorporating these scales in a complete way. These approaches are called multiscale methods. There are a large number of publications dedicated to multiscale problems and advances have been made in the simulation of large problems with highly heterogeneous rocks during the last two decades [8, 9, 10, 11, 12, 13, 14, 15, 16], in the context of different types of discretization models. Few multiscale methods consider the modeling of complex mechanisms (such as gravity segregation, and capillarity) [17].

In this direction, the goal of this work is to construct multiscale approximations for $2 \mathrm{D}$ and 3D reservoir two-phase flows on heterogeneous media under the effect of gravitational segregation, modeled by a system of equations with complex nonlinear interaction dynamics between the different physical effects. The novelty of the current approach is to prove the applicability of the Multiscale Hybrid Mixed finite element method, proposed and analyzed in [18, under the acronym MHM$\mathrm{H}$ (div), as the finite element model for the flux/pressure kernels embedded in the numerical model. The transport-hyperbolic problem is handled by a classical upstream differencing approach. Each time step is performed by an iterative coupling technique combined with a shared integration point memory implementation model.

The MHM-H(div) method adopts the Mixed Finite Element (MFE) formulation for flux/pressure discretization, where both variables are solved simultaneously. For subsurface applications, MFE models have found increasing acceptance over the years, since the pioneering work in [19]. For instance, they have been promoted by a research group at The University of Texas at Austin, USA, under Prof. M. Wheeler leadership, and by other groups (see e.g. [20, 21, 22, 23, 24, 25, 13, 26, 27, 28, 29, 30, 31, 32]). The success of this approach is mainly due to some relevant properties valid for these methods. For instance, it is well recognized that local mass conservation, continuous fluxes, and strong divergence-free enforcement for incompressible flows, which are crucial aspects for 
simulations based on realistic reservoir geology, are naturally verified by MFE methods. Moreover, there is a large flexibility in dealing with curved elements and well modeling, in the choice of their required approximation spaces, and in adaptivity designs for them. The convergence properties of MFE approximations are very well understood, the same optimal order for both the scalar pressure and vector flux variable being a common situation (with superconvergence occurring in some cases.

In the context of MFE formulations of Darcy's flows in porous media, the purpose of the introduction of a MHM principle is to cope with the complex geometry and inherent multiscale nature present in the modeled phenomena. The problem is divided into macro domains that facilitates the use of hierarchy of meshes and approximation spaces. The method makes use of coarse scale normal fluxes (trace variable) between subregions, the fine scale features inside each subregion being determined by resolving completely independent local Neumann problems, the boundary conditions being set by the trace variable. The MHM-H(div) method adopts the MFE formulation of the local Neumann problems, using flux and pressure representations by divergence-compatible FE pairs based on refined meshes inside the subregions. It should be observed that in the original MHM model [33], the proposal was to use $\mathrm{H}^{1}$-conforming local solvers (a version referred here as MHM- ${ }^{1}$ method).

Under some mild conditions on mesh and finite element space configuration (see Section 4.1), it was proved in [18] that the MHM-H(div) method can be interpreted as a classical MFE formulation of the model problem in the whole domain, based on a $\mathrm{H}(\mathrm{div})$-conforming space with normal components over the macro-partition interfaces constrained by the (less refined) trace space. Consequently, the relevant aspects of MFE methods for subsurface applications are inherited by the MHM-H(div) method. For instance, numerical verification tests presented in [18] revealed locally conservative, robust, and accurate results for the simulation of 2D and 3D Darcy's problems. These properties have also been demonstrated by MHM-H(div) flow simulations in 2D porous media with fractures [34], showing flexibility in the enforcement of the required coupling of two-dimensional matrix flow with the one-dimensional fracture flow. Moreover, the MHM-H(div) method can also be coupled with geomechanical deformations [35], where the elastic material properties are defined at the fine geocellular scales.

The MHM-H(div) method was the first MHM approach to provide error estimates using partitions of the domain by subregions of arbitrary polyhedral geometry, where quite general internal mesh refinement and/or polynomial degree increment are applied. Moreover, an efficient use of static condensation leads to a model order reduction, by solving flux and pressure only in terms of primary degrees of freedom associated to the trace variable and to the piecewise constant average pressure over each subregion, the fine flux and pressure details being resolved independently by the local Neumann problems. This procedure allows substantial reduction of the dominant computational costs associated with the flux/pressure kernel embedded in the numerical model.

The attempt to approximate nonlinear coupled multiphysics systems arising in reservoir simulations by solving the equations in the numerical model simultaneously is an approach that typically achieves unconditional stability and convergence. However, the price for robustness in these monolithic fully coupled methods is the construction and solution of large global fully coupled Jacobian systems, involving huge computational cost, and complicated code management. In this direction, a variety of sequential splitting schemes have been considered as optional approaches in order to increase the speed of computations. The principle is to decouple the whole system into separate subproblems, each one being constructed and solved separately in a sequence of intermediary stages during a time step, until a prescribed accuracy is reached. This strategy enables to tackle the complexity of the particular subproblem more efficiently. For the current context of fully coupled two-phase flow simulations, the proposal is to decouple the problem in a sequential fully implicit manner, adopting the MHM-H(div) method for the flux/pressure subproblem, and a finite volume transport solver. Thus, the resulting numerical model shares some similarities with the Sequential Fully Implicit (SFI) scheme of Multiscale Finite Volume methods [10, 36]. The reader can also find good review and recent developments of the SFI scheme in [37, 38], as well as of other developments on multiscale formulations in contrast to the approach considered in the current manuscript

Users should be aware that sequential methods do not necessarily guarantee unconditional stabil- 
ity and convergence, even though each uncoupled subproblem is unconditionally stable and convergent. For instance, for the cases of coupled fluid flow and reservoir geomechanic investigated in [39], SFI simulations are only conditionally stable when fixed-strain split schemes are used, while those with fixed-stress split schemes are unconditionally stable. For coupled flow and transport simulations (without mechanical influence), SFI strategies can be derived with convergence properties comparable with those of the fully implicit method, as discussed in [37, 38]. It should also be remarked that, in strong coupling situations, where sequential algorithms may face stability constraints, and for which an unconditionally stable monolithic fully coupled method is the recommended strategy, stabilized finite element methods [40, 41, 42, using a unified finite element simulator for all subsystems evolved, could be a helpful option.

\section{Outline of the paper}

Section 2 summarizes the mathematical models being considered. Section 3 presents the space and time discretizations as well the linearization scheme selected for the nonlinear equations and the sequentially fully implicit algorithm implemented. The main aspects of the MHM-H(div) strategy are described in Section 4. Two and three dimensional simulations are presented in Section 6, demonstrating the effectiveness of adopted approach. The numerical examples show that the proposed scheme is able to solve challenging coupled flow and transport problems. Concluding remarks, emphasizing the main aspects of the obtained results, are listed in Section 7 .

\section{Governing equations}

In this section the mathematical model is presented for two-phase flow, representing an isothermal immiscible oil-water system.

\section{$2.1 \quad$ Mathematical model}

The model is subject to the following hypotheses:

1. Dominant convective mass fluxes;

2. There is no chemical reaction, adsorption and precipitation;

3. Fluid flow is characterized by Darcy's law;

4. Injection and production wells are treated as boundary conditions;

5. Rock formation and fluids are incompressible;

6. Viscosity is constant;

7. Capillarity effects are neglected.

Relevant variables are the saturation $s_{\alpha}, \alpha \in\{w, o\}$ for oil and water, the total flux $\mathbf{q}$, and the pressure $p$ (unique for the two phases). The equations are written as the weighted pressure formulation presented in [43]. The strong formulation is a set of coupled equations expressed in terms of total flux and pressure for a elliptic sub-problem, and water saturation for a hyperbolic sub-problem. The problem is to find $\left(\mathbf{q}, p, s_{w}\right)$ verifying :

$$
\begin{gathered}
\left\{\begin{array}{ll}
(\lambda \mathbf{K})^{-1} \mathbf{q}+\nabla p-\mathbf{G}_{\lambda} & =0, \\
\nabla \cdot(\mathbf{q}) & =0,
\end{array} \text { in } \Omega \times[0, \mathrm{~T}],\right. \\
\phi \frac{\partial\left(s_{w}\right)}{\partial t}+\nabla \cdot\left(f_{w} \mathbf{q}+f_{w} f_{o} \mathbf{q}_{G w o}\right)=0, \quad \text { in } \Omega \times[0, \mathrm{~T}],
\end{gathered}
$$


where $\Omega$ is a polyhedral convex domain, $[0, T]$ is the time domain, $\mathbf{K}$ is the rock permeability tensor assumed to be symmetric and uniformly positive definite, and $\mathbf{G}_{\lambda}$ is the gravity term. Through the text, equations (1) and (2) are referred as flux/pressure and transport equations, respectively.

The functional relations and dependencies of the terms appearing in system (1)-(2) are presented in Table 1, where $\rho_{\alpha}, \eta_{\alpha}, f_{\alpha}$, and $\lambda_{\alpha}$ are the $\alpha$-phase mass density, dynamic viscosity, fractional flow and mobility. The porous medium is fully saturated with oil and water, i.e. $s_{w}+s_{o}=1$, and the gravity terms exhibit reciprocity, i.e. $\mathbf{q}_{G \alpha \beta}=-\mathbf{q}_{G \beta \alpha}$.

\begin{tabular}{|c|c|}
\hline Symbol & Expression \\
\hline $\mathbf{G}_{\lambda}\left(s_{w}, s_{o}\right)$ & $\left(f_{w} \rho_{w}+f_{o} \rho_{o}\right) \mathbf{g}$ \\
\hline$\lambda_{w}\left(s_{w}\right)$ & $\frac{k_{r w}}{n_{w}}$ \\
\hline$\lambda_{o}\left(s_{o}\right)$ & $\frac{k_{r_{o}}}{\eta_{o}}$ \\
\hline$\lambda\left(s_{w}, s_{o}\right)$ & $\lambda_{w}+\lambda_{o}$ \\
\hline$f_{\beta}\left(s_{\alpha}\right)$ & $\frac{\lambda_{\alpha}}{\lambda}$ \\
\hline $\mathbf{q}_{G \alpha \beta}\left(s_{w}, s_{o}\right)$ & $\mathbf{K} \lambda\left(\rho_{\alpha}-\rho_{\beta}\right) \mathbf{g}$ \\
\hline \multicolumn{2}{|c|}{ Darcy phase velocities } \\
\hline$\overline{\mathbf{v}}_{w}$ & $f_{w} \mathbf{q}+f_{w} f_{o} \mathbf{q}_{G w o}$ \\
\hline$\overline{\mathbf{v}}_{O}$ & $f_{o} \mathbf{q}+f_{o} f_{w} \mathbf{q}_{\text {Gow }}$ \\
\hline
\end{tabular}

Table 1: Relationships for the operators in (1) and (2). The subscript $\alpha, \beta \in\{o, w\}$, with $\alpha \neq \beta$.

The boundary $\partial \Omega$ is split into non-overlapping Dirichlet $\Gamma_{D}$ with non-zero measure and Neumann $\Gamma_{N}$ parts. Thus, the boundary conditions for the pressure equation are

$$
\begin{aligned}
\mathbf{q} \cdot \mathbf{n}=0, & \text { on } \Gamma_{N} \times[0, \mathrm{~T}], \\
p=p_{D}, & \text { on } \Gamma_{D} \times[0, \mathrm{~T}],
\end{aligned}
$$

where $\mathbf{n}=\mathbf{n}^{\Omega}$ is the outward oriented unitary normal vector to $\partial \Omega$. The boundary condition for the transport equation is defined only at the inlet upwind boundary with non-zero measure $\Gamma_{i n}$,

$$
s_{w}=s_{w_{i n}}, \quad \text { on } \Gamma_{i n} \times[0, \mathrm{~T}] .
$$

To close the mathematical model, consider the initial condition

$$
s_{w}(\mathbf{x}, t=0)=s_{w}^{0}, \quad \text { in } \quad \Omega .
$$

\section{The sequential multiscale finite element approach}

The adopted discrete model uses spatial discretization combining MHM-H(div) method for the flux/pressure equations, and the finite volume (FV) method for the transport equation. The sequential fully implicit (SFI) approach is employed as a solver for the nonlinear approximated equations.

\subsection{Discretization}

In this section, the full discretization for equations (1) and (2) is introduced. Throughout this paper, for a region $\mathcal{R} \subseteq \Omega$, scalar and vector Hilbert spaces $L^{2}(\mathcal{R})$ and $\mathrm{H}^{s}(\mathcal{R})$ have the usual meaning and norms. The space $\mathrm{H}(\operatorname{div}, \mathcal{R})$ is composed by square-integrable vector functions, for which the divergence is also square integrable. The notation $(\cdot, \cdot)_{\mathcal{R}}$ is used for the $L^{2}$-inner products, and $\langle\cdot, \cdot\rangle_{\partial \mathcal{R}}$ refers to the duality pairing between $\mathrm{H}^{1 / 2}(\partial \mathcal{R})$ and $\mathrm{H}^{-1 / 2}(\partial \mathcal{R})$. The subscript $\mathcal{R}$ will be dropped if $\mathcal{R}=\Omega$.

Discrete time instants $0=t^{0}<\ldots<t^{N}=T$ are considered over the time domain $[0, \mathrm{~T}]$ with uniform time step size $\Delta t$, and let the approximate variables at $t^{n}=n \Delta t$ be indexed as $\left(\mathbf{q}, p, s_{w}\right)^{n}=\left(\mathbf{q}^{n}, p^{n}, s_{w}^{n}\right)$. Partitions $\mathcal{T}$ of the computational domain are also considered for the construction of finite element approximation spaces. 


\section{Flux/pressure kernel}

The MFE formulation for the system (1) is based on approximation spaces:

$$
\begin{aligned}
& \mathscr{V} \subset H_{0}(\operatorname{div}, \Omega)=\left\{\boldsymbol{\tau} \in H(\operatorname{div}, \Omega) ;\left.\boldsymbol{\tau} \cdot \mathbf{n}^{\Omega}\right|_{\Gamma_{N}}=0\right\}, \\
& \mathscr{P} \subset L^{2}(\Omega),
\end{aligned}
$$

piece-wise defined over a partition $\mathcal{T}$, verifying the divergence-compatibility property $\nabla \cdot \mathscr{V} \subset \mathscr{P}$. Thus, the mixed problem to be solved is to find the pair $(\mathbf{q}, p)^{n+1} \in \mathscr{V} \times \mathscr{P}$ at time $t^{n+1}$ such that

$$
\left\{\begin{array}{l}
\left(\left(\mathbf{K} \lambda^{n+1}\right)^{-1} \mathbf{q}^{n+1}, \boldsymbol{\tau}\right)-\left(p^{n+1}, \nabla \cdot \boldsymbol{\tau}\right)=-\left\langle\boldsymbol{\tau} \mathbf{n}^{\Omega}, p_{D}\right\rangle_{\Gamma_{D}}+\left(\mathbf{G}_{\lambda}^{n+1}, \boldsymbol{\tau}\right), \quad \forall \boldsymbol{\tau} \in \mathscr{V}, \\
\left(\nabla \cdot \mathbf{q}^{n+1}, \varphi\right)=0, \quad \forall \varphi \in \mathscr{P} .
\end{array}\right.
$$

where $\lambda^{n+1}=\lambda\left(s_{w}^{n+1}\right)$ and $\mathbf{G}_{\lambda}^{n+1}=\mathbf{G}_{\lambda}\left(s_{w}^{n+1}\right)$. It is important to note that the problem above is linear in $(\mathbf{q}, p)^{n+1}$.

\section{Transport equation}

The selected functional space for the saturation is $\mathscr{P}_{0}(\mathcal{T})$, consisting of piece-wise constant functions over each element $K \in \mathcal{T}$. The discrete finite volume (FV) formulation for the transport equation (2) searches $s_{w}^{n+1} \in \mathscr{P}_{0}(\mathcal{T})$ verifying

$$
\left(\phi s_{w}^{n+1}, v\right)+\Delta t\left\langle\mathcal{F}\left(s_{w}^{n+1}, \mathbf{q}^{n+1}\right), v\right\rangle_{\partial K \backslash \Gamma_{i n}}=\left(\phi s_{w}^{n}, v\right)-\Delta t\left\langle s_{w_{i n}} \mathbf{q}^{n+1} \cdot \mathbf{n}, v\right\rangle_{\Gamma_{i n}},
$$

$\forall v \in \mathscr{P}_{0}(\mathcal{T})$. The nonlinear numerical flux function over $\partial K$ is expressed in the form

$$
\mathcal{F}\left(s_{w}^{n+1}, \mathbf{q}^{n+1}\right)=\left[f_{w}\left(s_{w}^{n+1}\right) \mathbf{q}^{n+1}+f_{w} f_{o}\left(s_{w}^{n+1}\right) \mathbf{q}_{G w o}\left(s_{w}^{n+1}\right)\right] \cdot \mathbf{n},
$$

The problem 7 is approximated by the first-order upwind scheme. Regarding the treatment for the numerical fluxes, there are several upwinding schemes, we review two of them. The first one is described in [44], which is in essence an explicit scheme, but it can be reformulated as an implicit scheme. In its implicit form is referred to as Phase Potential Upwinding (PPU) [45]. This approach has slow convergence mainly because for a fixed total flow rate, the potential upwinding leads to oscillations and consequently the divergence for the nonlinear solver. The second approach is implicit in its original form and separates the effects from the viscous and the gravity flow rates. It is referred to as Implicit Hybrid Upwinding (IHU) [38. Such a scheme provides a numerical flux that is monotonic in the cell saturation and eliminates the oscillations present in the PPU. Heretofore, we adopt the second one, and the validity for its implementation is provided later. During the solution of the discrete equation (7) water transport is implicitly solved, allowing the use of relatively large time steps, and resulting in a robust implementation. The drawback of using first order upwind schemes is that the saturation profiles are diffuse. A multirate time integration scheme can be used to control the error during the transport evolution [17, but it is out of the scope of this manuscript.

\subsection{Solving the equations sequentially}

As mentioned in the Introduction, SFI algorithms are common approaches to handle nonlinear multiphysics coupled equations typically occurring in reservoir simulations. The principle is to split the system into different subproblems, and to solve separately each subproblem using specialized efficient implicit solvers [38]. Here, SFI techniques are considered for approximations of the coupled system (6) and (7) in conjunction with a MHM-H(div) approach for the pressure system.

Given approximations $\left(\mathbf{q}, p, s_{w}\right)^{n}$ at instant $t^{n}$, the SFI algorithm provides the approximate solution $\left(\mathbf{q}, p, s_{w}\right)^{n+1}$ at the next time step by applying an external loop with counter $m$ and the maximum number of iterations $m_{s f i}$, executing a sequence of two solvers, a linear solver for the pressure/flux equations and a nonlinear solver for the transport equation, leading to the intermediary approximate 
solutions $\left(\tilde{\mathbf{q}}, \tilde{p}, \tilde{s}_{w}\right)^{m}$ respectively. Information transfer between both solvers is performed using integration point operations. The state of $\tilde{s}_{w}^{m}$ is transferred to the pressure equation solver to compute the new pair $(\tilde{\mathbf{q}}, \tilde{p})^{m+1}$ considering no variation in saturation. Then, $\tilde{\mathbf{q}}^{m+1}$ is transferred to the transport equation solver and a new $\tilde{s}_{w}^{m+1}$ is computed considering no variation in flux. The sequence is repeated until a desired stopping criterion is reached as a function of the variation of both sets of variables.

Algorithm 1 summarizes the main steps of the SFI computations. Some details on the linearization scheme for the transport equation and on the information transfer operations are given in the following subsections. The MHM-H(div) scheme adopted to solve the pressure system is described in Section 4 .

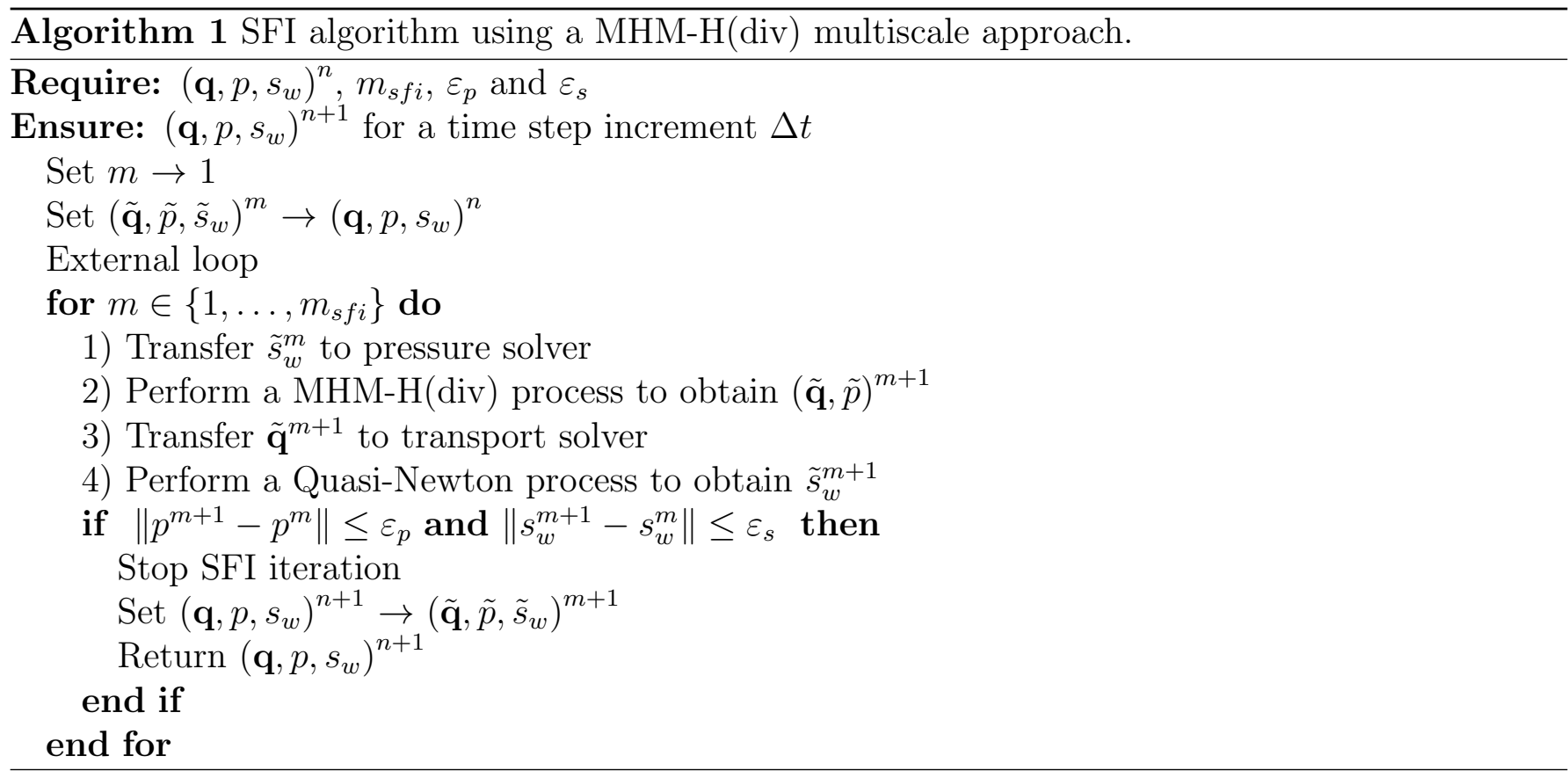

\subsubsection{Linearization scheme for the transport equation}

The use of the Newton method as linearization scheme for the transport equations results in a low computational performance and a lack of convergence for the algorithm [38]. Quasi-Newton methods give lower convergence rates but have lower cost of execution time. There are several options when Quasi-Newton approaches are adopted, as documented in [46, 47, 48]. In this research, a QuasiNewton strategy for the step 4 in algorithm 1 which is inspired on the ideas of Sloan documented in [49]. In general, given the nonlinear problem $F(\mathbf{x})=0$ to be solved, the Quasi-Newton iteration is defined as:

$$
\begin{cases}\delta \mathbf{x}_{Q N}\left(\mathbf{x}^{k-1}\right) & =-\nabla \tilde{F}^{-1} F\left(\mathbf{x}^{k-1}\right) \\ \mathbf{x}^{k} & =\mathbf{x}^{k-1}+\delta \mathbf{x}_{Q N}\left(\mathbf{x}^{k-1}\right)\end{cases}
$$

where $\tilde{F}$ is an approximation for the Jacobian matrix of $F$. For the two-phase equations, we proposed that $\tilde{F}$ is taken as the matrix computed with linear relative permeability at each time step. When this approach is adopted, the nonlinear solver is stable and convergent, but possesses low convergence rates. The convergence rate can be enhanced by considering a simple nonlinear acceleration technique. In [50] was introduced an accelerated convergence for the Newton method employing a two-step iterative method. In that iterative method, a first step is to perform a second-order Newton step to evaluate a iteration function $\psi$ for a subsequent state(s), in short $\mathbf{x}^{k}=\psi\left(\mathbf{x}^{k-1}\right)$. The second move is to perform another Newton step that combines the information of a subsequent state(s) and the value of the iterative function $\psi\left(\mathrm{x}^{k-1}\right)$ leading to better convergence. The ideas of [51] and [49] are combined to define a form of the function $\psi\left(\mathrm{x}^{k-1}\right)$ leading to a Quasi-Newton method QN, and the modified Thomas method for the transport problem QNT. Details for them are provided concisely. 
Method QN A new state $\mathbf{y}^{k}$ is provided by the iteration function:

$$
\mathbf{y}^{k}=\psi\left(\mathbf{x}^{k-1}\right)=\mathbf{x}^{k-1}+\delta \mathbf{x}_{Q N}\left(\mathbf{x}^{k-1}\right)
$$

A simple Quasi-Newton's method is:

$$
\mathbf{x}^{k}=\mathbf{y}^{k}
$$

This method requires one assembly, linear solve, and one function evaluation per update.

Method QNT This method defines the iteration function as follows:

$$
\mathbf{y}^{k}=\psi\left(\mathbf{x}^{k-1}\right)=\mathbf{x}^{k-1}+\omega^{k-1} \delta \mathbf{x}_{Q N}\left(\mathbf{x}^{k-1}\right)
$$

With the new update state defined as follows:

$$
\mathbf{x}^{k}=\mathbf{y}^{k}+\omega^{k-1} \delta \mathbf{x}_{Q N}\left(\mathbf{y}^{k}\right)
$$

Where the factor $\omega$ is the so-called acceleration factor [49], defined as :

$$
\omega^{k}=\omega^{k-1}+\frac{\delta \mathbf{x}_{Q N}\left(\mathbf{x}^{k-1}\right) \cdot \delta \mathbf{x}_{Q N}\left(\mathbf{y}^{k}\right)}{\delta \mathbf{x}_{Q N}\left(\mathbf{x}^{k-1}\right) \cdot \delta \mathbf{x}_{Q N}\left(\mathbf{x}^{k-1}\right)}
$$

The initial acceleration factor $\omega^{0}$ is set to 1.0 at the beginning of the iterative process. This method provides a better convergence but requires two assemblies, two linear solve and two function evaluation per update, i.e. two Quasi-Newton steps. Different high order methods are provided by [51] with varying extra evaluations of the function and system inversions. For the method QNT, the enhanced convergence is shown later in the numerical results section.

\section{MHM-H(div) method for flux/pressure systems}

In this section, we highlight the main aspects of the MHM-H(div) method to be applied in the flux/pressure kernel arising in the discretization of the two-phase flow described in the previous section. The assumptions on the partitions of the computational domain $\Omega$ and on the two-scale FE approximation spaces based on them to be used, the local-global set of equations to be solved, and the adopted implementation strategy is described here. This method was proposed in [18], where a unified general error analysis is presented for all the considered two-scale space scenarios.

\subsection{Setting the main assumptions}

For the MHM multiscale formulation, the finite-dimensional approximation spaces are based on a macro partition $\mathcal{T}^{0}=\left\{\Omega_{i}\right\}$ of the computational domain $\Omega_{i}$. The sub-domains $\Omega_{i}$ may have one of the usual element geometry, but the focus is on more general polyhedral subdomains (which may even be non-convex). Associated to $\mathcal{T}^{0}$, let the mesh skeleton $\Gamma$ be formed by the union of all faces $F \subset \partial \Omega_{i} \backslash \partial \Omega_{N}$. A fixed vector field $\mathbf{n}$ is defined such that $\left.\mathbf{n}\right|_{F}$ is a vector normal to an internal face $F \subset \Gamma$, and for $F \subset \partial \Omega$.

Following [18], a two-scale hierarchy of meshes and FE spaces are constructed. The parameters $\gamma=\left(\gamma_{s k}, \gamma_{i n}\right)$ are used to indicate coarse and refined mesh widths and polynomial degrees, which are assumed to be suitable for accurate simulation of the problem at hand:

- $\gamma_{s k}=\left(h_{s k}, k_{s k}\right)$ correspond to a coarse trace space $\Lambda_{\gamma_{s k}}$ defined over $\Gamma$.

- $\gamma_{i n}=\left(h_{i n}, k_{i n}\right)$ are the parameters for the local FE spaces defined in the interior of the subregions $\Omega_{i}$.

They are obtained by the following steps: 
1. Coarsest one-scale setting:

- There is a coarse conformal shape regular partition $\mathcal{T}_{h_{s k}}$ of $\Omega$, formed by the union of coarse sub-meshes $\mathcal{T}_{h_{s k}}^{\Omega_{i}}$, with characteristic size $h_{s k}$. A partition $\mathcal{T}_{h_{s k}}^{\Gamma}$ is induced over $\Gamma$ by $\mathcal{T}_{h_{s k}}$.

- FE spaces $\mathcal{E}_{\gamma_{s k}}=\mathscr{V}_{\gamma_{s k}} \times \mathscr{P}_{\gamma_{s k}} \subset H_{0}(\operatorname{div}, \Omega) \times \subset L^{2}(\Omega)$ are defined by local FE pairs $\mathscr{V}_{\gamma_{s k}}\left(\Omega_{i}\right) \times \mathscr{P}_{\gamma_{s k}}\left(\Omega_{i}\right) \subset H\left(\operatorname{div}, \Omega_{i}\right) \times L^{2}\left(\Omega_{i}\right)$, based on $\mathcal{T}_{h_{s k}}^{\Omega_{i}}$, which are divergence-compatible: $\nabla \cdot \mathscr{V}_{\gamma_{s k}}\left(\Omega_{i}\right)=\mathscr{P}_{\gamma_{s k}}\left(\Omega_{i}\right)$.

- A trace space $\Lambda_{\gamma_{s k}}$, based on $\mathcal{T}_{h_{s k}}^{\Gamma}$, is defined by functions $\left.\mu\right|_{F}=\left.\boldsymbol{\tau} \cdot \mathbf{n}\right|_{F}$, , for $F \subset \partial \Omega_{i} \backslash \partial \Omega_{N}$, and $\boldsymbol{\tau} \in \mathscr{V}_{\gamma_{s k}}\left(\Omega_{i}\right)$. They are piecewise scalar polynomials of degree $k_{s k} \geq 0$.

2. Fine-scale local space settings:

- Refined internal partitions $\mathcal{T}_{h_{i n}}^{\Omega_{i}}$ are obtained by subdivision of $\mathcal{T}_{h_{s k}}^{\Omega_{i}}$. Suppose $h_{i n} \sim h_{s k} / 2^{\ell}$, $\ell \geq 0$.

- Local divergence-compatible FE pairs $\mathcal{E}_{\gamma_{i n}}\left(\Omega_{i}\right)=\mathscr{V}_{\gamma_{i n}}\left(\Omega_{i}\right) \times \mathscr{P}_{\gamma_{i n}}\left(\Omega_{i}\right) \subset H\left(\operatorname{div}, \Omega_{i}\right) \times$ $L^{2}\left(\Omega_{i}\right)$, based on $\mathcal{T}_{h_{i n}}^{\Omega_{i}}$, are considered for $k_{i n}=k_{s k}+n, n \geq 0$.

- By construction, $\mathscr{V}_{\gamma_{s k}}\left(\Omega_{i}\right) \subset \mathscr{V}_{\gamma_{i n}}\left(\Omega_{i}\right)$, so that the trace embedding property holds:

$$
\Lambda_{\gamma_{s k}} \subset \Lambda_{\gamma_{i n}}=\left\{\mu ;\left.\mu\right|_{\partial \Omega_{i} \backslash \partial \Omega_{N}}=\boldsymbol{\tau} \cdot \mathbf{n}, \boldsymbol{\tau} \in \mathscr{V}_{\gamma_{i n}}\left(\Omega_{i}\right)\right\}
$$

3. Two-scale setting:

- The MHM-H(div) method to be considered is for FE divergence-compatible pairs $\mathcal{E}_{\gamma}\left(\Omega_{i}\right)=$ $\mathscr{V}_{\gamma}\left(\Omega_{i}\right) \times \mathscr{P}_{\gamma_{i n}}\left(\Omega_{i}\right) \subset H\left(\operatorname{div}, \Omega_{i}\right) \times \subset L^{2}\left(\Omega_{i}\right)$, where

$$
\mathscr{V}_{\gamma}\left(\Omega_{i}\right)=\left\{\boldsymbol{\tau} \in \mathscr{V}_{\gamma_{i n}}\left(\Omega_{i}\right) ;\left.\boldsymbol{\tau} \cdot \mathbf{n}\right|_{\partial \Omega_{i} \backslash \partial \Omega_{N}} \in \Lambda_{\gamma_{s k}}\right\}
$$

Notice that the trace constraint (14) is the guaranty for the well definition of the flux spaces 15). Moreover, the direct sum decomposition $\mathscr{V}_{\gamma}\left(\Omega_{i}\right)=\mathscr{V}_{\gamma_{s k}}^{\partial}\left(\Omega_{i}\right) \oplus \mathscr{\mathscr { V }}_{\gamma_{i n}}\left(\Omega_{i}\right)$ holds in terms of the trace component $\mathscr{V}_{\gamma_{s k}}^{\partial}\left(\Omega_{i}\right) \subset \mathscr{V}_{\gamma_{s k}}\left(\Omega_{i}\right)$, and the internal component ${\stackrel{\mathscr{V}}{\gamma_{i n}}}\left(\Omega_{i}\right) \subset \mathscr{V}_{\gamma_{i n}}\left(\Omega_{i}\right)$, with normal traces vanishing over $\partial \Omega_{i}$. The pressure spaces can also be decomposed as $\mathscr{P}_{\gamma_{i n}}\left(\Omega_{i}\right)=\mathscr{P}_{0}\left(\Omega_{i}\right)+\mathscr{P}_{\gamma_{i n}}^{\perp}\left(\Omega_{i}\right)$, the functions in $\mathscr{P}_{0}\left(\Omega_{i}\right)$ being constant in $\Omega_{i}$, and $\mathscr{P}_{\gamma_{i n}}^{\perp}\left(\Omega_{i}\right)$ denoting the $L^{2}$-orthogonal complement of $\mathscr{P}_{\gamma_{i n}}^{\perp}\left(\Omega_{i}\right)$ in $\mathscr{P}_{\gamma_{i n}}\left(\Omega_{i}\right)$.
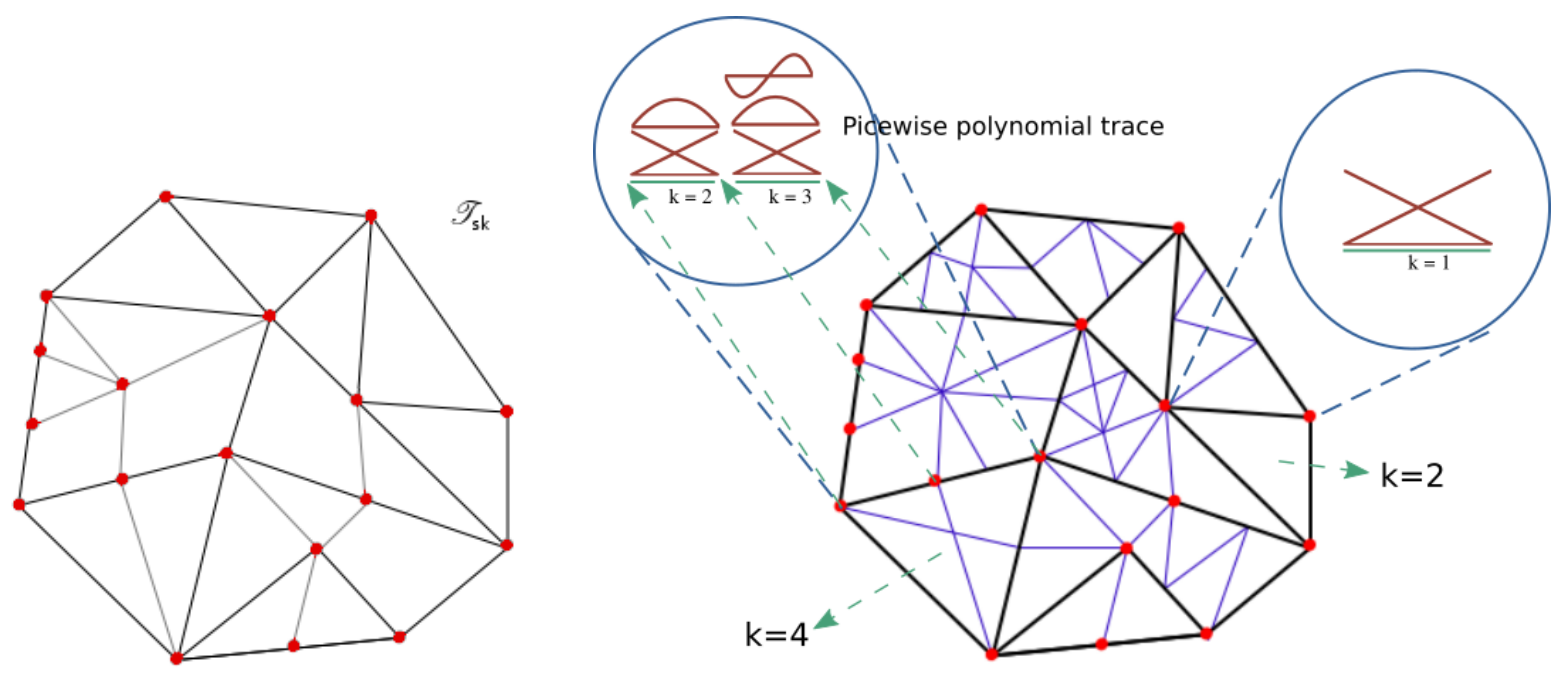

Figure 1: Diagram illustration of some aspects of MHM-H(div) partitions and FE space configurations. 
Some aspects of the partitions and FE space configurations for the MHM-H(div) method are illustrated in Figure 1. A coarse conformal partition $\mathcal{T}_{h_{s k}}$ inducing $\mathcal{T}_{h_{s k}}^{\Gamma}$ over $\Gamma$ is shown in the left hand side, and local refined partitions $\mathcal{T}_{h_{i n}}^{\Omega_{i}}$ are constructed by subdivisions of the partitions $\mathcal{T}_{h_{s k}}^{\Omega_{i}}$, as shown in right hand side. Observe that over a face $F=\Omega_{i} \cap \Omega_{j}$, the meshes $\mathcal{T}_{h_{i n}}^{\Omega_{i}}$ and $\mathcal{T}_{h_{i n}}^{\Omega_{j}}$ may be non-conformal. The kind of polynomial spaces verifying trace space hierarchy that can be used for the MHM-H(div) method is also illustrated, indicating that the polynomial degree distribution over the subdomains may be non-uniform. However, for the well definition of the method, the constraint (14) needs to be satisfied.

\subsection{The method}

Consider the two-scale pair $\mathcal{E}_{\gamma}=\mathscr{V}_{\gamma} \times \mathscr{P}_{\gamma_{i n}}$, where

$$
\begin{aligned}
\mathscr{V}_{\gamma} & =\left\{\boldsymbol{\tau} \in H(\operatorname{div}, \Omega) ;\left.\boldsymbol{\tau}\right|_{\Omega_{i}} \in \mathscr{V}_{\gamma}\left(\Omega_{i}\right),\left.\boldsymbol{\tau} \cdot \mathbf{n}^{\Omega}\right|_{\partial \Omega_{N}}=0\right\} \\
\mathscr{P}_{\gamma_{i n}} & =\left\{\varphi \in L^{2}(\Omega) ;\left.\varphi\right|_{\Omega_{i}} \in \mathscr{P}_{\gamma_{i n}}\left(\Omega_{i}\right)\right\} .
\end{aligned}
$$

Recall that the divergence-compatibility property $\nabla \cdot \mathscr{V}_{\gamma}=\mathscr{P}_{\gamma_{i n}}$ is verified, and the interface normal trace constraint $\left.\boldsymbol{\tau} \cdot \mathbf{n}\right|_{\Gamma} \in \Lambda_{\gamma_{s k}}$ holds for all $\boldsymbol{\tau} \in \mathscr{V}_{\gamma}$. The problems of interest in this paper, to be solved in each iteration of the SFI algorithm described in Section 2 have the form: find $(\tilde{\mathbf{q}}, \tilde{p}) \in \mathcal{E}_{\gamma}$ satisfying

$$
\left\{\begin{array}{l}
\left(\mathbf{A}^{-1} \tilde{\mathbf{q}}, \boldsymbol{\tau}\right)-(\tilde{p}, \nabla \cdot \boldsymbol{\tau})=-\left\langle\boldsymbol{\tau} \mathbf{n}^{\Omega}, p_{D}\right\rangle_{\Gamma_{D}}+(\mathbf{b}, \boldsymbol{\tau}), \quad \forall \boldsymbol{\tau} \in \mathscr{V}_{\gamma} \\
(\nabla \cdot \tilde{\mathbf{q}}, \varphi)=0, \quad \forall \varphi \in \mathscr{P}_{\gamma_{i n}}, .
\end{array}\right.
$$

where $\mathbf{A}=\mathbf{K} \lambda$ and $\mathbf{b}=\mathbf{G}_{\lambda}$.

\subsubsection{Local-global scheme}

The MHM-H(div) method can be interpreted as a scheme to compute $(\tilde{\mathbf{q}}, \tilde{p})$ by local-global algorithms, by exploiting the hierarchical structure of the FE space setting $\mathcal{E}_{\gamma}$. It is designed in the spirit of the primal MHM-H ${ }^{1}$ technique [33], both methods sharing the following characteristics:

(i) The local-global algorithms are discrete versions of a local-global characterization of the exact solution at the continuous level.

(ii) A new normal flux variable $\tilde{\sigma} \in \Lambda_{\gamma_{s k}}$ is introduced, and an orthogonal decomposition is set for pressure, $\tilde{p}=\tilde{p}_{0}+\tilde{p}^{\perp}$, where $\tilde{p}_{0} \in \mathscr{P}_{0}(\mathcal{T}) \subset \mathscr{P}_{\gamma_{i n}}$ is piecewise constant over $\mathcal{T}$, and $\tilde{p}^{\perp} \in \mathscr{P}_{\gamma_{\text {in }}}^{\perp}$ is in $L^{2}$-orthogonal complement of $\mathscr{P}_{0}(\mathcal{T})$ in $\mathscr{P}_{\gamma_{i n}}$.

(iii) In the first coarse scale level, $\tilde{\sigma} \in \Lambda_{\gamma_{s k}}$ and $\tilde{p}_{0} \in \mathscr{P}_{0}\left(\mathcal{T}^{0}\right)$ are computed by a stable global system (upscaling stage).

(iv) The second fine scale, detailed components of the solution $\tilde{\mathbf{q}} \in \mathscr{V}_{\gamma}$ and $\tilde{p}^{\perp} \in \mathscr{P}_{\gamma_{\text {in }}}^{\perp}$ are computed by solving a set of completely independent problems, each one restricted to a subregion $\Omega_{i} \in \mathcal{T}$, using $\tilde{\sigma}$ as Neumann boundary data over $\partial \Omega_{i}$ (downscaling stage).

The well posed downscaling and upscaling stages of the MHM-H(div) method are defined as follows:

\section{Downscaling stage: local boundary value Neumann problems}

Giving $\tilde{\sigma} \in \Lambda_{\gamma_{s k}}$, the downscaling stage consists in finding $\tilde{\mathbf{q}}=\tilde{T}^{\mathbf{q}}(\tilde{\sigma}) \in \mathscr{V}_{\gamma}$ and $\tilde{p}^{\perp}=\tilde{T}^{p}(\tilde{\sigma}) \in \mathscr{P}_{\gamma_{i n}}^{\perp}$ satisfying the following Neumann boundary problem in each subregion $\Omega_{i}$ :

$$
\left\{\begin{array}{l}
\left(\mathbf{A}^{-1} \tilde{T}^{\mathbf{q}}(\tilde{\sigma}), \stackrel{\circ}{\mathbf{v}}\right)_{\Omega_{i}}-\left(\tilde{T}^{p}(\tilde{\sigma}), \nabla \cdot \stackrel{\circ}{\mathbf{v}}\right)_{\Omega_{i}}=(\mathbf{b}, \stackrel{\circ}{\mathbf{v}}), \quad \forall \stackrel{\circ}{\mathbf{v}} \in \mathscr{\mathscr { V }}_{\gamma_{i n}}\left(\Omega_{i}\right), \\
\left(\nabla \cdot \tilde{T}^{\mathbf{q}}(\tilde{\sigma}), \varphi^{\perp}\right)_{\Omega_{i}}=0 \quad \forall \varphi^{\perp} \in \mathscr{P}_{\gamma_{i n}}^{\perp}\left(\Omega_{i}\right), \\
\left.\tilde{T}^{\mathbf{q}}(\tilde{\sigma}) \cdot \mathbf{n}\right|_{\partial \Omega_{i} \backslash \partial \Omega_{N}}=\left.\tilde{\sigma}\right|_{\partial \Omega_{i} \backslash \partial \Omega_{N}} .
\end{array}\right.
$$




\section{Upscaling stage: global problem}

The upscaling stage consists of finding $\tilde{\sigma} \in \Lambda_{\gamma_{s k}}$ and $\tilde{p}_{0} \in \mathscr{P}_{0}\left(\mathcal{T}^{0}\right)$ such that for all $\mu \in \Lambda_{\gamma_{s k}}$, and $v_{0} \in \mathscr{P}_{0}\left(\mathcal{T}^{0}\right)$,

$$
\left\{\begin{array}{l}
\left(\mathbf{A}^{-1} \tilde{T}^{\mathbf{q}}(\tilde{\sigma}), \tilde{T}^{\mathbf{q}}(\mu)\right)-\left(\tilde{p}_{0}, \nabla \cdot \tilde{T}^{\mathbf{q}}(\mu)\right)=\left(\mathbf{b}, \tilde{T}^{\mathbf{q}}(\mu)\right)-<\mu, p_{D}>_{\partial \Omega_{D}}, \forall \mu \in \Lambda_{\gamma_{s k}}, \\
\left(\nabla \cdot \tilde{T}^{\mathbf{q}}(\tilde{\sigma}), v_{0}\right)=0, \forall v_{0} \in \mathscr{P}_{0}\left(\mathcal{T}^{0}\right)
\end{array}\right.
$$

\subsubsection{Remarks}

As briefly mentioned in the Introduction, there are two major issues that qualify the MHM-H(div) method as a stable, accurate and efficient option for the flux/pressure kernel occurring in each time step.

1. The MHM-H(div) can be interpreted as a well posed mixed formulation of Darcy's problems in the whole domain, based on the $H(\mathrm{div})$-conforming space $\mathscr{V}_{\gamma}$ with normal components over the macro-partition interfaces constrained by the trace space $\Lambda_{\gamma_{s k}}$, and showing divergence compatibility with the pressure space $\mathscr{P}_{\gamma_{i n}}$, as shown in [18, Theorem 1]. Using this characterization, MHM-H(div) method inherits the positive properties of MFE methods. For instance:

- Classical tools for mixed methods can be used for convergence analysis of MHM-H(div) solutions. For instance, in [18, Theorem 4] a unified error analysis is valid for meshes and space configurations as general as the ones described in Section 4.1. Depending on the choice of the two-scale mesh and polynomial degree parameters $\left(h_{s k}, k_{s k}\right)$ and $\left(h_{i n}, k_{i n}\right)$, optimal convergence rates in the natural norms are obtained. For instance, for sufficiently smooth solutions approximated by Raviart-Thomas space configurations for tetrahedral or for hexahedral local meshes $\mathcal{T}_{h_{i n}}^{\Omega_{i}}$,

$$
\begin{aligned}
\| \mathbf{q}-\left.\tilde{\mathbf{q}}\right|_{\mathbf{L}^{2}(\Omega)} & =O\left(h_{s k}^{k_{s k}+1}\right), \\
\|\nabla \cdot(\mathbf{q}-\tilde{\mathbf{q}})\|_{L^{2}(\Omega)} & =O\left(h_{i n}^{k_{i n}+1}\right), \\
\|p-\tilde{p}\|_{L^{2}(\Omega)} & =O\left(h_{s k}^{k_{s k}+2}\right)+O\left(h_{i n}^{k_{i n}+1}\right) .
\end{aligned}
$$

Notice that the flux variable is approximated with the same accuracy order $O\left(h_{s k}^{k_{s k}+1}\right)$ as for the trace variable, and super-convergence $O\left(h_{i n}^{k_{i n}+1}\right)$ for the divergence of the flux. Concerning the errors for pressure, the two terms on the right hand side may have different influence on the results. Without polynomial degree enrichment $k_{i n}=k_{s k}=k$, the second term becomes dominant for less refined internal meshes. For instance, when $h_{s k}=h_{i n}=$ $h$ it gives the standard well known convergence rate $O\left(h^{k+1}\right)$, as for the flux variable. However, when $k_{i n}>k_{s k}$, the influence of second term reduces as compared with the first one, independently of internal mesh refinement, to produce enhanced pressure convergence rate $O\left(h_{s k}^{k_{s k}+2}\right)$.

- Local mass conservative velocities are observed at the micro-scale elements inside the subregions, an essential property for accurate transport of multi-species in heterogeneous media [23].

- For incompressible flows, the resulting flux is strongly divergence-free, due to the divergencecompatibility condition.

2. Downscaling-upscaling stages, as the one occurring in the MHM-H(div) method, are crucial for the construction of efficient computational multiscale algorithms. They offer the possibility of decomposing the resolution of the problem in terms of expensive, but independent, local solvers that can profit from parallel strategies, and cheaper coupled global systems. In the particular MHM-H(div) context, the adopted algorithms correspond to standard static condensation procedures [52], which can be performed at two computational cycles, as described in [18]: 
- The first level cycle is applied to assemble and solve the condensed global problem (static condensation on macro-subregions $\Omega_{i} \in \mathcal{T}^{0}$ ). Representing the action of the upscaling operation, it considers as primary variables the face fluxes $\tilde{\sigma} \in \Lambda_{\gamma_{s k}}$ over the macro-mesh skeleton and $\tilde{p}_{0} \in \mathscr{P}_{0}\left(\mathcal{T}^{0}\right)$ of one pressure DoF per macro-subregion. It also provides the downscaling operation for the computation of the secondary internal solutions inside macro elements.

- The second level cycle (optional) is employed for order model reduction of the local MFE Neumann solvers occurring in the downscaling operation (static condensation on micro elements $K \in \mathcal{T}^{\Omega_{i}}$ ), using similar principles as for the first level cycle.

It can be proved that MHM- $\mathrm{H}^{1}$ and $\mathrm{MHM}-\mathrm{H}(\mathrm{div})$ methods are derived from equivalent localglobal characterizations of the exact solution in infinite-dimensional spaces. However, their discrete finite dimensional versions differ in some specific aspects:

- The MHM- $\mathrm{H}^{1}$ formulation uses $\mathrm{H}^{1}$ - conforming downscaling local solvers to compute $\tilde{p}^{\perp}$ in the interior of the subdomains, whilst the mixed formulation is used in the MHM-H(div) method to determine the variables $\tilde{p}^{\perp}$ and $\tilde{\mathbf{q}}$. Consequently, the way the Neumann boundary conditions are imposed by the local solvers differ: for the MHM-H(div) formulation the normal flux coming from the fine-scale inside $\Omega_{i}$ is strongly enforced and constrained to $\tilde{\sigma} \in \Lambda_{\gamma_{s k}}$ at $\partial \Omega_{i}$, as expressed in the third equation in the system (19). Conversely, Neumann boundary conditions are imposed by the MHM- $\mathrm{H}^{1}$ formulation in a weak multiplier sense.

- Local mass conservative velocities at the micro elements and strongly enforcement of the divergence free constraint are not properties typical of $\mathrm{H}^{1}$-conforming solvers. Moreover, permeability anisotropy furnishes a challenge for these discretization methods. Thus, these difficulties are faced by the MHM- $\mathrm{H}^{1}$ method as well, specially for less refined discretizations. As already mentioned, they are not a concern in MFE methods [23], as is the case of the MHM-H(div) formulation.

- Both MHM schemes also differ in their implementations. For the MHM-H ${ }^{1}$ simulations reported in the literature, the implementation consists in pre-computing especial multiscale shape functions determined by the action of the downscaling stage on a given set of basic functions for $\Lambda_{\gamma_{s k}}$. Then, they are used to assemble the global condensed system of the upscaling stage. Notice that similar approaches are also adopted in other multiscale methods, as summarized in [31], and could be used for the MHM-H(div) implementations as well. However, the static condensation strategy adopted by MHM-H(div) implementations, as described previously in Remark 2, the computation of multiscale basis functions is not required.

\section{Computational aspects}

In the following, we provide a brief discussion on the computational aspects of the method. As is presented in algorithm 1, it is required the execution for steps 1 to 4 . Steps 1 and 3 are dedicated to the information transfer between the modules. In step 2, a proper separation between the static and dynamic information during the multiscale process can be performed to achieve higher performance. Regarding step 4, the nonlinear acceleration technique adopted allows to reduce transport iterations.

\section{1 offline-online computations}

In the following, we provide a brief discussion of the computational aspects of the method. As is presented in algorithm 1, it is required the execution for steps 1 to 4 . Steps 1 and 3 are dedicated to the information transfer between the modules. In step 2, a proper separation between the static and dynamic information during the multiscale process can be performed to achieve higher performance. Regarding step 4, the nonlinear acceleration technique adopted allows reducing transport iterations. 
In this research, no computational $\mathrm{CPU}$ is reported because the main goal is to demonstrate the capability of our approach in solving two-phase flows.

\section{Offline:}

There is an overhead regarding all the upscaling and downscaling steps. The intensive computations at this level are:

- Transferring the static reservoir properties like $\mathbf{K}(\mathbf{x})$ and $\phi(\mathbf{x})$;

- The computation for the internal problems associated with the MHM-H(div).

For the computation of an external properties map, we use intensively the approach given in [53] for geometric search. Queries like the identification of a voxel in a rasterized porosity and permeability maps are required to populate each the finite element / finite volume at the centroid as the sample point. Once the problem geometry is defined, the interpolation of the field data can be performed once and save in disk the computation object. For the reader interested in the complexity of such an approach can be referred to 53 .

In the MHM-H(div) idealization the internal fields are computed by a set of local Neumann problems with mixed finite elements. Each subproblem leads to the construction in parallel for a sparse matrix. All these matrix objects are collectively connected by a local transmission problem, which requires the identification of the skeleton fluxes and a macro element-wise static condensation [18]. It is a very intensive CPU operation that can be performed in parallel and once by providing the geometry partition. In other words, all the statically condensed matrices are stored and saved to be used during the online phase. Because the total mobility $\lambda$ is constant per microelement, the condensed system per microelement can be updated by inexpensive matrix blocks multiplications for a given $\lambda$ value.

\section{Online:}

The overhead in this phase is only attached to the amount of output to be reported per time step. The intensive computations at this level are:

- The assembly of the condensed MHM-H(div) problem;

- The nonlinear solution process for the transport equation.

For the assembly of the global transmission problem, we take advantage of that the total mobility $\lambda$ is constant and after the update of the pre-computed micro-element matrices, the MHM-H(div) global transmission problem is obtained without the need to recompute every micro-element matrix during the solution process for the MHM-H(div).

Regarding eh transport equation, the execution for a Quasi-Newton step is performed in parallel, and in combination with the nonlinear acceleration, we obtain an efficient nonlinear solver for the transport module.

\subsection{Information transfer between modules}

The implementation of the SFI method offers modular programming and allows to select a proper numerical approach for generating approximations for both the pressure and transport equations. However, the SFI algorithm requires two transfer operations for the saturation and the flux between the equations at each stage (see steps 1 and 3 in algorithm 1). A simple shared memory management interface is applied to perform the transfer of information. The information transfer depends on the following rules: 
- The geometric mesh resolution inside the subdomains is the same for both, the flux/pressure kernel and transport discretizations.

- The finite elements should have the same indexation, between the pressure and transport discretization at the fine scale.

- There is no need to reconstruct normal flux traces required for numerical flux computations used in the finite volume scheme of the transport problem, for they are directly provided by the MHM-H(div) solver of the flux/pressure kernel.

- In the flux/pressure system only $\lambda$ and $\mathbf{G}_{\lambda}$ depend on the saturation, requiring saturation transfer from the transport solution during time evolution.

In step 1, saturation and fluxes are stored in computational vector objects associated with the centroid of the elements, such that in a single parallel for all the saturation are transferred. In step 3, the normal flux integral is transferred to the transport element interfaces. A linear sparse application is created to transfer the normal flux by a simple sparse-matrix- vector multiplication.

\section{$6 \quad$ Numerical Results}

The described multiscale method for two-phase flows is now incorporated in the multiphysics finite element NeoPZ computational framework1. As emphasized in [54], the NeoPZ users can implement numerical techniques where each physical phenomenon or scale component is approximated by its most appropriate numerical scheme, a crucial property for the implementation of the method considered in the current manuscript.

To evaluate the combination of the MHM-H(div) approach with the SFI algorithm, several cases are explored with a heterogeneous porosity distribution. For the simulation of complex geological geometries, the use of a mesh generator is required. In this research a Gmsh oriented script with CAD support was developed for the generation of the geometry.

In this section several numerical results are presented. They are introduced to show different characteristics of the computational implementation.

1. Gravitational segregation: Two cases are included to show the correctness for the implemented IHU fluxes;

2. Water flooding 2D: This case plots the well representation as boundaries and the effect of the skeleton approximation is studied;

3. Water flooding 3D: This case shows the capability for the proposed method for dealing with complex geometries and heterogeneous rock formations.

Table 2 shows the common parameters among all the numerical presented tests. In addition, because we use the Euler method only linear mixed finite elements are considered for the entire section.

\begin{tabular}{|c|c|c|c|c|c|c|c|}
\hline$p_{i}$ & $p_{p}$ & $g$ & $\rho_{g}$ & $\rho_{w}$ & $k_{r \alpha}$ & $k_{r \beta}$ & $\epsilon_{s}=\epsilon_{p}$ \\
\hline \hline $25 \mathrm{MPa}$ & $10 \mathrm{MPa}$ & $9.81 \mathrm{~m} \mathrm{~s}^{-2}$ & $800 \mathrm{~kg} \mathrm{~m}^{-3}$ & $1000 \mathrm{~kg} \mathrm{~m}^{-3}$ & $s_{\alpha}^{2}$ & $\left(1-s_{\beta}\right)^{2}$ & $1.0 \times 10^{-6}$ \\
\hline
\end{tabular}

Table 2: Common parameters for all the simulations.

\footnotetext{
${ }^{1}$ NeoPZ open source platform: http://github.com/labmec/neopz
} 


\subsection{Gravitational segregation examples}

In the following, two different examples showing the correctness for the gravity fluxes are provided. The first one is related to the vertical gravitational segregation of carbon dioxide (CO2) migrating vertically through a porous column. The second one is a synthetic case that shows the gravitational effect on a pure two-dimensional configuration.

\subsubsection{CO2 segregation in a vertical column}

The two-phase incompressible flow of fluids water and CO2 (super-critical conditions) in a porous medium can be described by the system of equations introduced previously. In this case the light phase is $\mathrm{CO} 2$ and the heavy phase is the water. The column is $10[\mathrm{~m}]$ and the parameters for the simulation are presented in table 3 .

The flux function has a bell shape shown in figure 2 (black line). The construction of a semianalytic solution is provided [55]. They construct semi-exact solutions for a set of different configurations in the material parameters: homogeneous; piecewise homogeneous; layered periodic and finally fully heterogeneous. For the sake of simplicity, we just cover the homogeneous case to show the approximation properties of our finite volume scheme.

The Riemann problem can be approximated by a semi-analytic solution in space and time. The initial saturation of $\mathrm{CO} 2$ is $s_{0}=0.8$ (see figure 3 (Gray line)). Figure 2 shows relevant points for the construction for the semi-exact solution. Table 3 shows the parameters used for the simulation. As can be observed in figure 3 , the velocity of the $\operatorname{Shock}\left(s_{3}\right)$ is greater than the velocity of the $\operatorname{Shock}\left(s_{1}\right)$, indicating that the plume spreads. As $\operatorname{Shock}\left(s_{1}\right)$ and $\operatorname{Shock}\left(s_{2}\right)$ propagate in opposite directions, the area of the $\mathrm{CO} 2$ plume saturated with $s_{0}=0.8$, contracts as time increases and it disappears when $\operatorname{Shock}\left(s_{1}\right)$ and Shock $\left(s_{2}\right)$ meet together. We can remark the good agreement between semi-analytic solution and the simulation. Figure 3 documents the correctness for the implementation for IHU fluxes accounting for the gravity effect. In addition, 3 can be observed a good agreement for the approximated solution and the semi-exact.

\begin{tabular}{|c|c|c|c|c|c|c|c|}
\hline$\rho_{g}$ & $\rho_{w}$ & $\mu_{g}=\mu_{w}$ & $\kappa$ & $\phi$ & $t_{f}$ & $h$ & $\epsilon_{s}=\epsilon_{p}$ \\
\hline \hline $700 \mathrm{~kg} \mathrm{~m}^{-3}$ & $1000 \mathrm{~kg} \mathrm{~m}^{-3}$ & $0.001 \mathrm{Pas}$ & $1.0 \times 10^{-12} \mathrm{~m}^{2}$ & 0.1 & $1.0 \mathrm{~d}$ & $10 \mathrm{~m}$ & $1.0 \times 10^{-6}$ \\
\hline
\end{tabular}

Table 3: Parameters for the vertical column. 


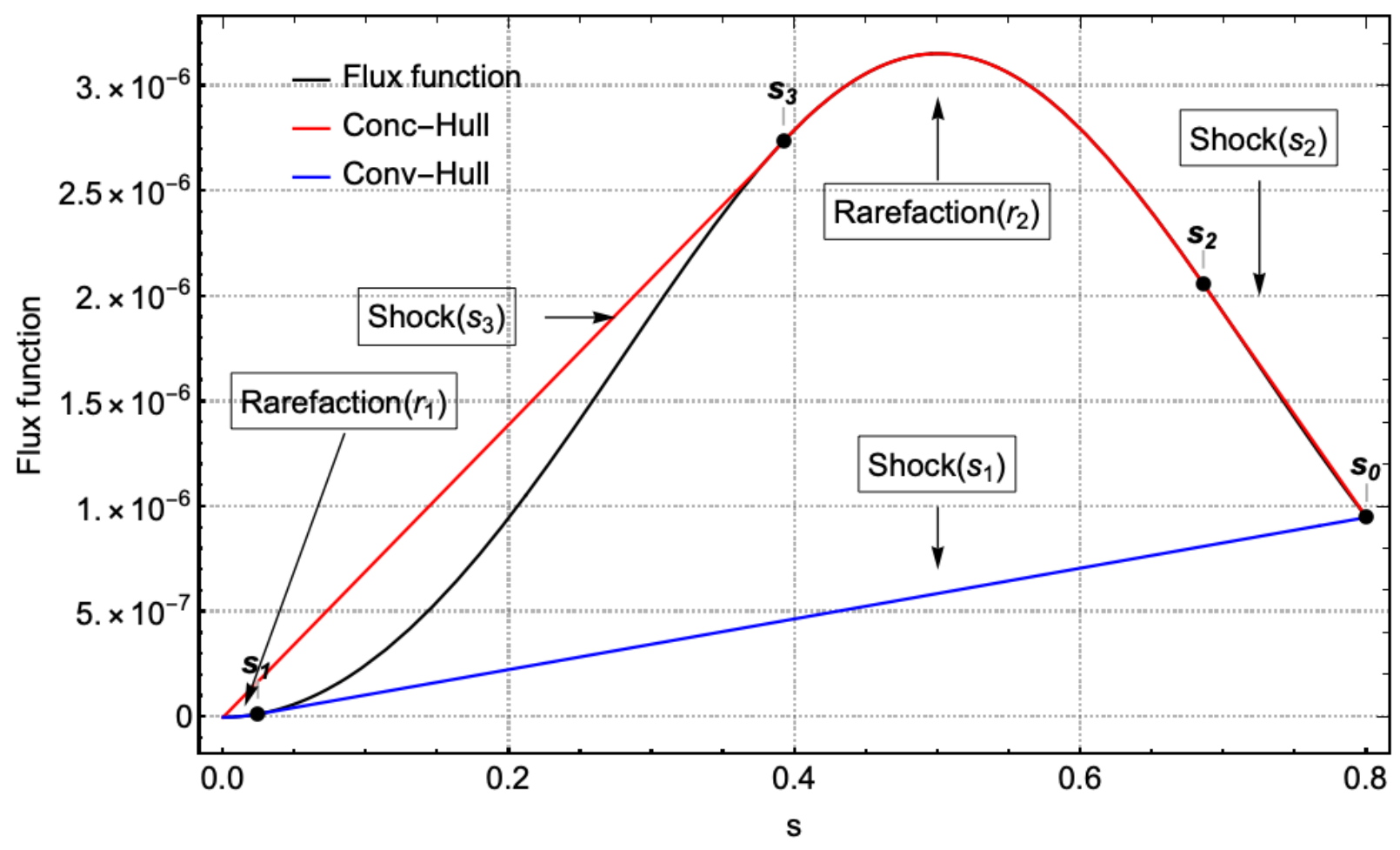

Figure 2: Vertical column: Construction of the convex and concave hulls [55].
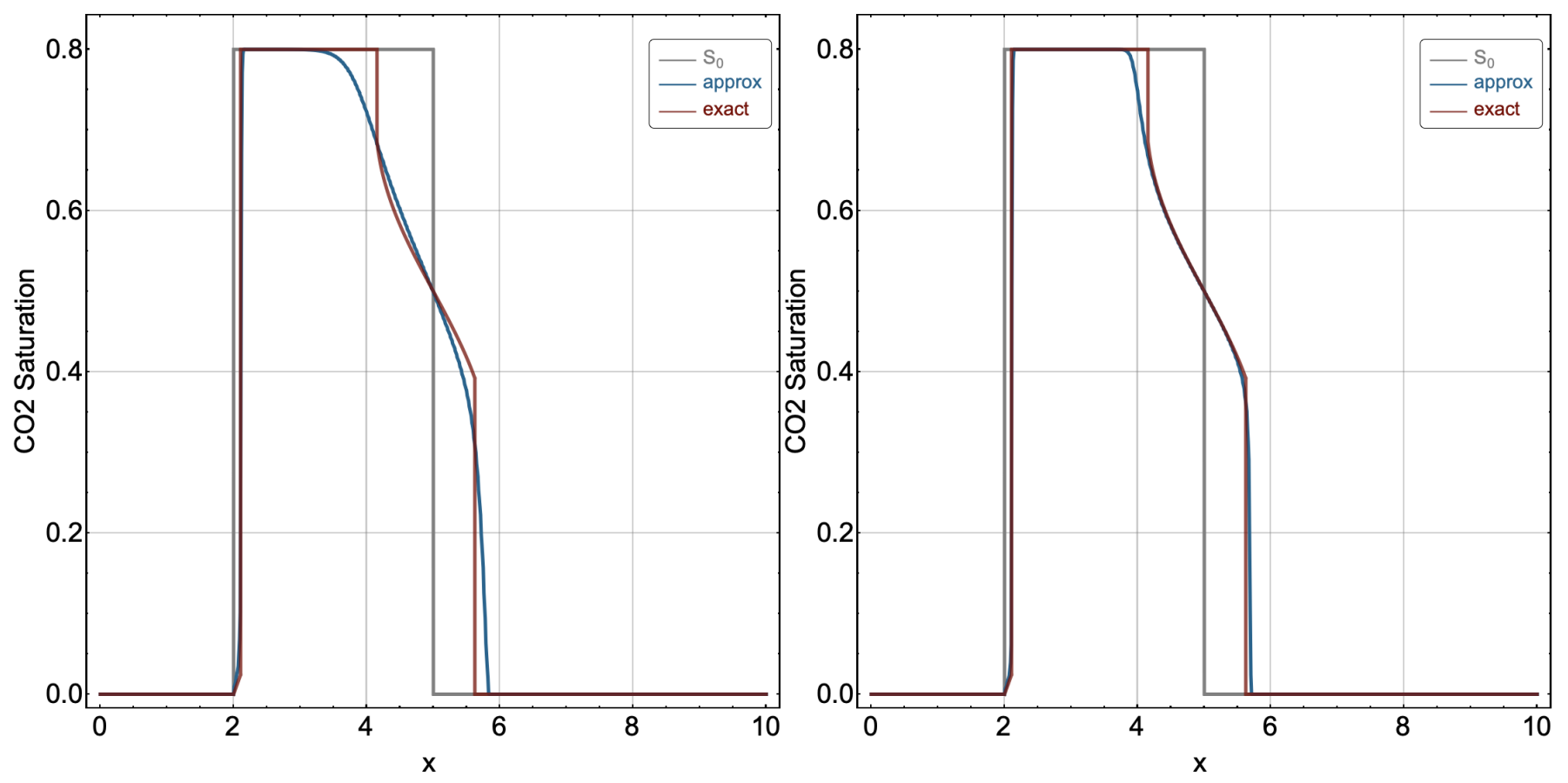

Figure 3: Vertical column: Approximation at $t=1[$ day $] . \Delta t=0.1$ (left-side) and $\Delta t=0.025$ (right-side).

\subsubsection{Water segregation in squared domain}

Let us consider the two-dimensional problem in $\left(0, t_{f}\right) \times \Omega, \Omega=(0,10) \times(0,10)$. It is considered quadrangular meshes obtained by splitting each cell into four with the same size (uniform refinement), giving elements with size $h=2^{-l}$ with $l \in\{0,1,2\}$. Table 4 shows the parameters used for the simulation. The initial water saturation is distributed using a level set function 21 with $r=0.25$ and 
$c=10$ (see figure 5 for $t=0$ ).

$$
f=-r^{2}+(x-5)^{2}+(y-5)^{2}-c
$$

\begin{tabular}{|c|c|c|c|c|c|c|}
\hline$\mu_{w}=\mu_{o}$ & $\kappa$ & $\phi$ & $t_{f}$ & $\Delta t$ & $h$ & $s_{0}$ \\
\hline \hline $0.001 \mathrm{Pas}$ & $1.0 \times 10^{-13} \mathrm{~m}^{2}$ & 0.1 & $100.0 \mathrm{~d}$ & $1.0 \mathrm{~d}$ & $10 \mathrm{~m}$ & 1.0 \\
\hline
\end{tabular}

Table 4: Parameters for the segregation in squared domain.

In this case, we vary the SFI tolerances as $\epsilon_{s}=\epsilon_{p}=1^{-l} l \in\{7,8,9,10\}$ for the stop criterion. We monitor the integral of the water in the domain along the simulation time. The objective is to provide numerical evidences of that mass loss is controlled naturally by the number of SFI iterations. Figure 4 on the left side shows that as the SFI tolerance decreases, the mass loss decreases in the same order. Indeed for this case the mass loss is of order $\mathcal{O}\left(\epsilon_{s}\right)$.

Figure 4 on the right side shows that the cost of having small mass loss carries larger number of SFI iterations. However, this issue can be addressed by some nonlinear acceleration techniques [38, 56, 32. Figure 5 shows the evolution of the water segregated by the effect of gravity at times values $t=\{0,25,50,100\} \mathrm{d}$.
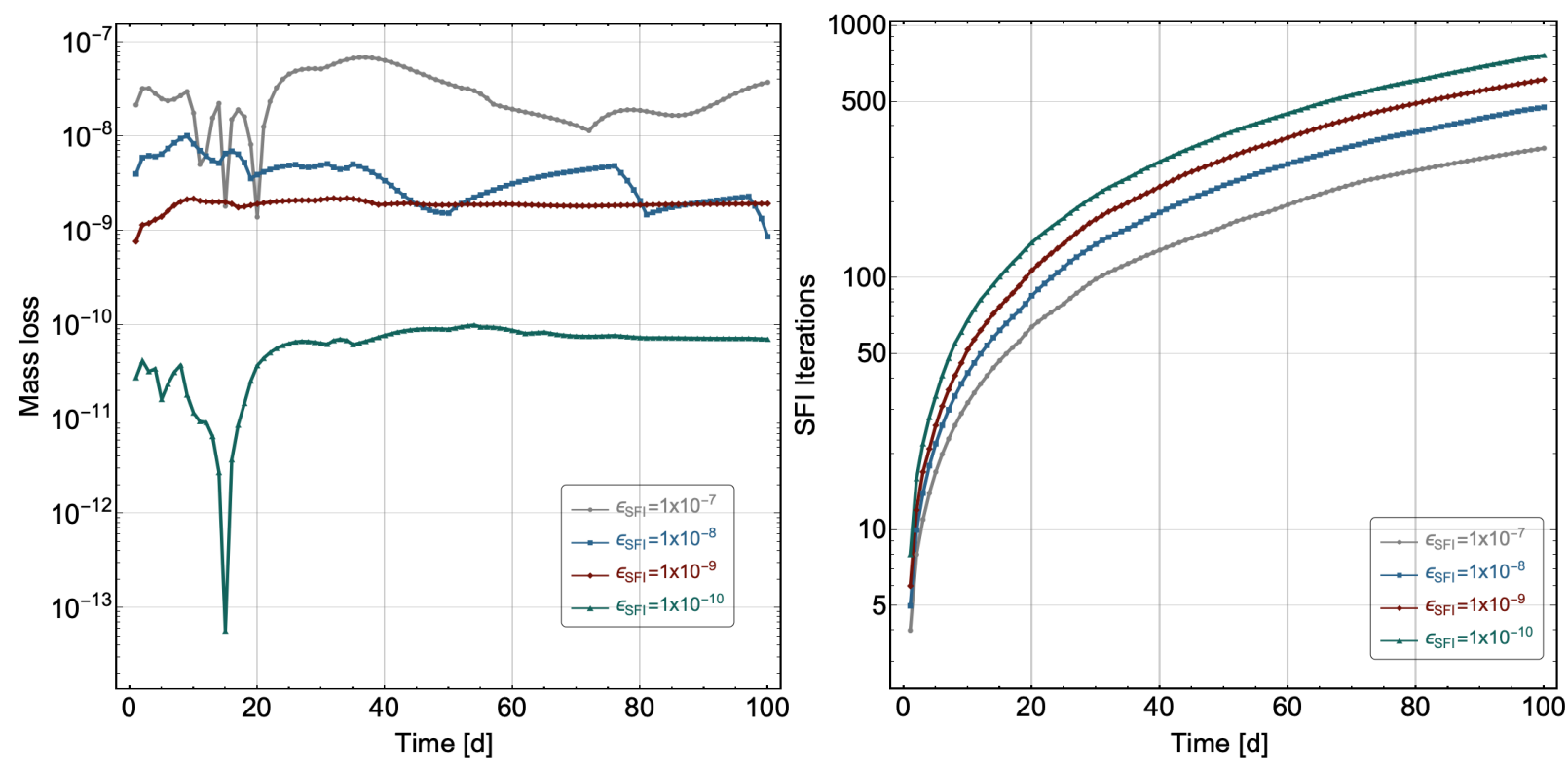

Figure 4: Gravitational segregation: Mass loos and SFI iterations v.s. simulation time. 

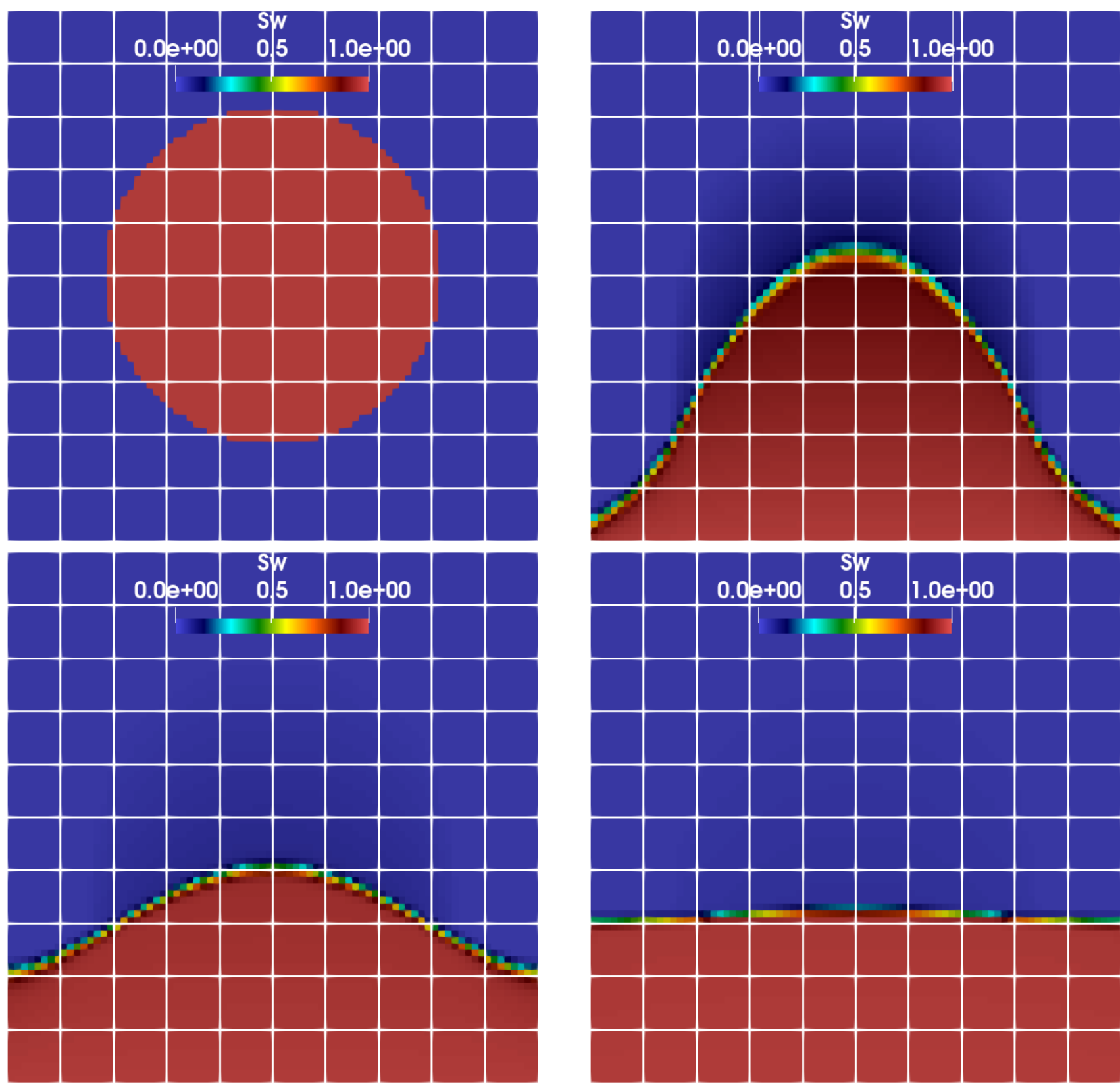

Figure 5: Gravitational segregation: Water saturation distribution at $t=\{0,25,50,100\}$ d. The white wireframe represents the skeleton mesh for the MHM-Hdiv operator.

\subsection{Water flooding 2D}

The two-dimensional problem in $\left(0, t_{f}\right) \times \Omega, \Omega=(-500,500) \times(-50,50)$. The physical configuration is represented by two horizontal injectors at reservoir flanks and one producer in the center of the geometry. Three different levels of refinement in the skeleton mesh are considered $l=\{0,1,2\}$. Figure 6 shows the geometry and the skeleton mesh (cyan wireframe representation) used for the computations.

Next, figure 7 shows the permeability and the porosity transferred to the mesh elements and 5 presents the simulation parameters.

\begin{tabular}{|c|c|c|c|c|c|}
\hline$\mu_{w}$ & $\mu_{o}$ & $t_{f}$ & $\Delta t$ & $s_{0}$ & $\epsilon_{s}$ \\
\hline \hline $0.001 \mathrm{Pas}$ & $0.002 \mathrm{Pas}$ & $4000.0 \mathrm{~d}$ & $20.0 \mathrm{~d}$ & 0.0 & $1.0 \times 10^{-4}$ \\
\hline
\end{tabular}

Table 5: Parameters of the water flooding. 


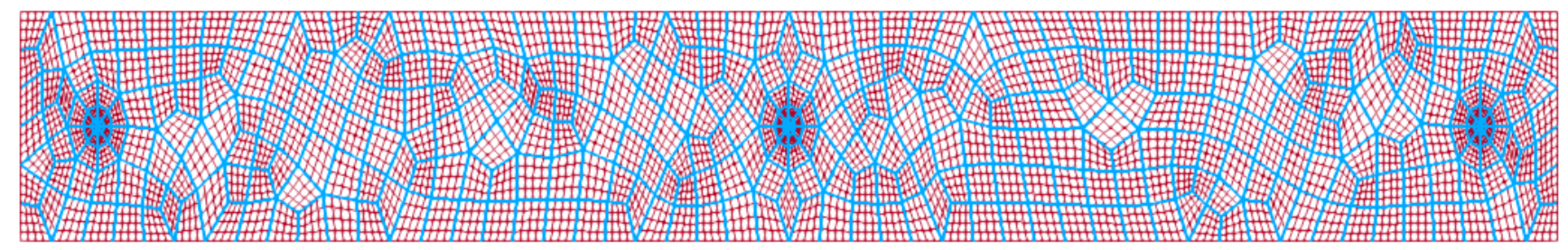

Figure 6: Water flooding: Unstructured fine partition (in red) used to compute the reference solution of the 2D water flooding problem; the coarsest macro subregions are represented in cyan color.
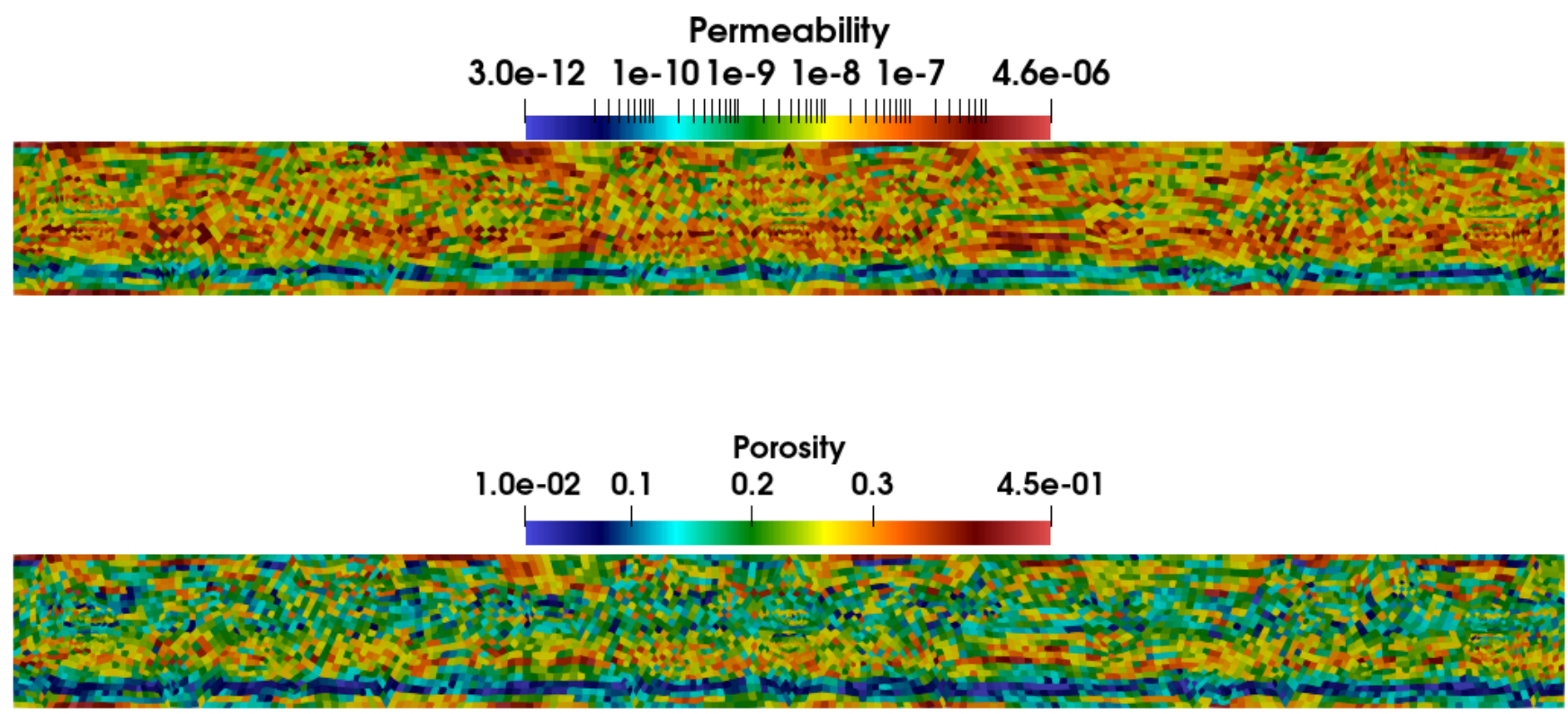

Figure 7: Water flooding: Permeability and porosity maps transferred to the fine scale mesh.

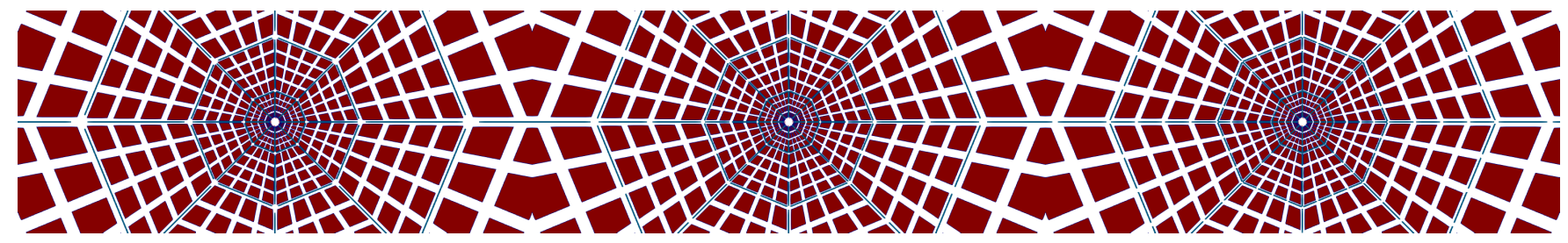

Figure 8: Water flooding: Details on skeleton refinement near producer wellbore. $l=0$ (top-side), $l=1$ (mid-side), and $l=2$ (bottom-side).

Figure 8 shows details for the skeleton refinement for each level $l=0$ (left-side) $l=1$ (mid-side) and $l=2$ reference configuration (right-side). It is important to remark the mesh is adapted towards the wellbore in order to capture properly the multiscale details near the wellbore region. 


\begin{tabular}{|c|c|c|c|}
\hline Setting & DoF & DoF (SC) & $\%$ \\
\hline \hline MHM-H (div), $l=0$ & 108312 & 2064 & 1.91 \\
\hline MHM-H (div), $l=1$ & 109936 & 3688 & 3.35 \\
\hline Reference & 113184 & 6936 & 6.13 \\
\hline
\end{tabular}

Table 6: Water flooding: Number of equations in the condensed linear systems (DoF) for MHM-H (div) and reference mixed simulation.
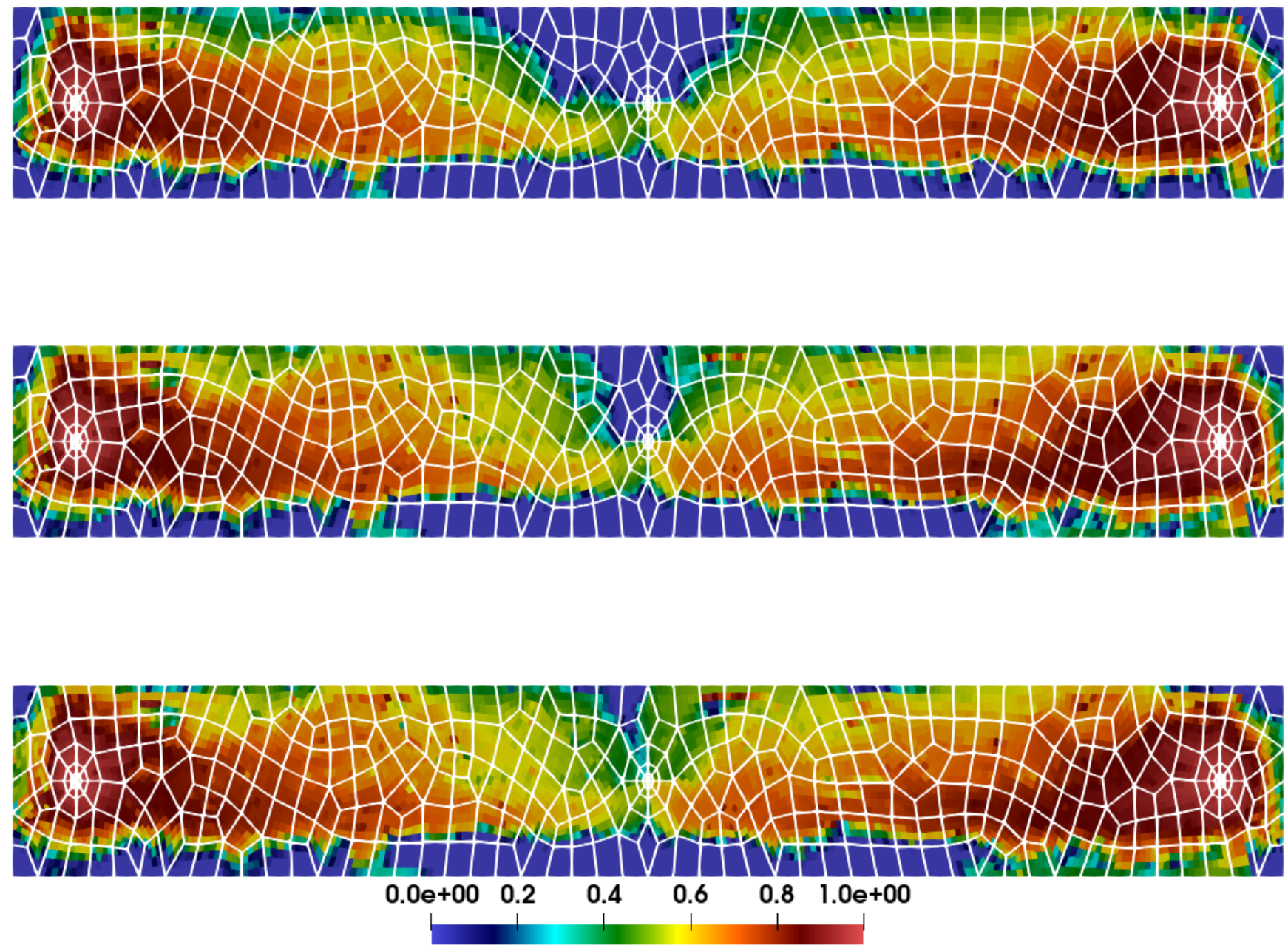

Figure 9: Water flooding: water saturation fields at instant $t_{f}$, using the MHM- H(div) method at levels $l=\{0,1\}$, and the reference approximation. In each case, the mesh skeleton is represented by the white wireframe.

Table 6 shows the number of equations, condensed equations and the percentage of the equation being active in the global transmission problem. During the online phase, it shows the pay-off for the static condensation procedure [18].

Figure 10 (left-side) shows that comparison for the pressure approximations plotted over the line $(\{-500,0\},\{0,500\})$. The main characteristics are approximated by the case $l=1$ in comparison with the reference solution $l=2$ (Single-scale simulation). 

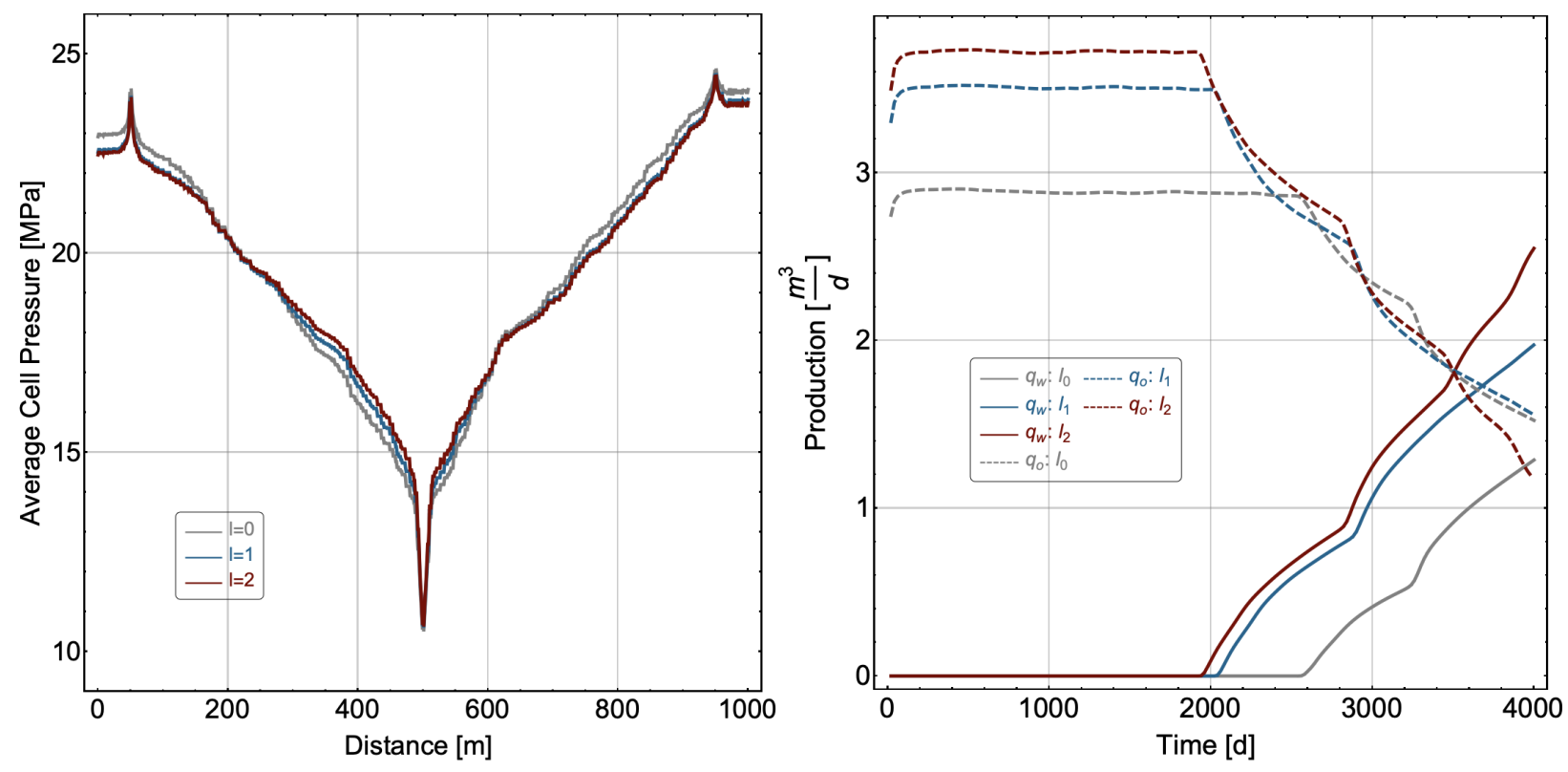

Figure 10: Water flooding: water saturation fields at instant $t_{f}$, using the MHM-H(div) method at levels $l=\{0,1\}$, and the reference approximation (Red line).

The figure 10 (right-side) shows the water (continuous line) and oil production (dashed lines) for $l=\{0,1\}$ and the reference solution (red lines). The coarse skeleton approximation $l=0$ induces a larger error in the normal flux used to solve the transport equation, it leads to a substantial difference in the break-through time. As well in the figure 9 (top-side) the saturation map for $l=0$ has a big difference in comparison with the others solutions. For the case of $l=1$, the flux approximation is more accurate and the difference with the reference solution is smaller. The water break-through time in those simulations is around $2000 \mathrm{~d}$. Figure 9 (mid-side) can be observed that in general the approximation captures the main features for the reference solution (bottom-side).

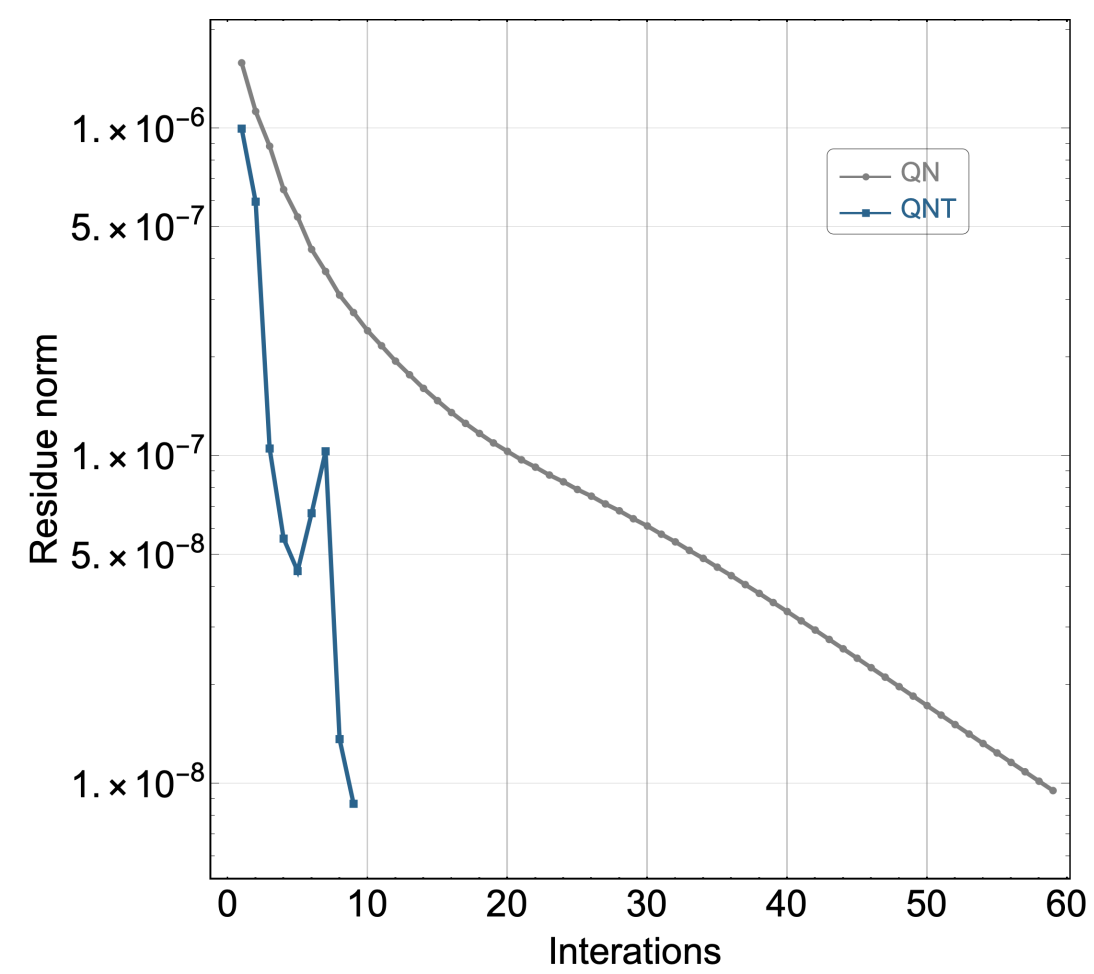

Figure 11: Water flooding: Transport module iterations for a single SFI iteration.

Figure 11 documents the acceleration obtained in the transport module by considering the QNT method. To reach the same value for the residue expression the QN uses 59 iterations while the QNT 
only spends 9 iterations.

\subsection{Synthetic reservoir 3D}

Unisim I-D is a synthetic reservoir model based on the "Namorado" field located in Campos Basin in Brazil. This model was developed and described in [57]. In this research, the UNISIM-I-D model has been simplified to the two-phase case (oil-water system) with no faults inside the reservoir and quadratic relative permeability model. The physical configuration is composed of 3 vertical injection wells placed at reservoir flanks and two vertical producers in the central region. Table 7 shows the parameters used for the 3D case.

\begin{tabular}{|c|c|c|c|c|c|c|}
\hline$\mu_{w}$ & $\mu_{o}$ & $t_{f}$ & $\Delta t$ & $h$ & $s_{0}$ & $\epsilon_{s}=\epsilon_{p}$ \\
\hline \hline $0.001 \mathrm{Pas}$ & $0.002 \mathrm{Pas}$ & $12000.0 \mathrm{~d}$ & $10.0 \mathrm{~d}$ & $100 \mathrm{~m}$ & 0.0 & $1.0 \times 10^{-4}$ \\
\hline
\end{tabular}

Table 7: Parameters of the synthetic reservoir model.

The geometry was pre-processed from the layers horizons and meshed in an unstructured way. Figure 12 shows the reservoir geometry on the left side and the corresponding areal view on the right side. The permeabilities and porosities are obtained from the data set available on https: //www. unisim.cepetro.unicamp.br/benchmarks/en/unisim-i/unisim-i-d. Figure 13 shows the permeability and porosity element constant-wise fields being transferred.
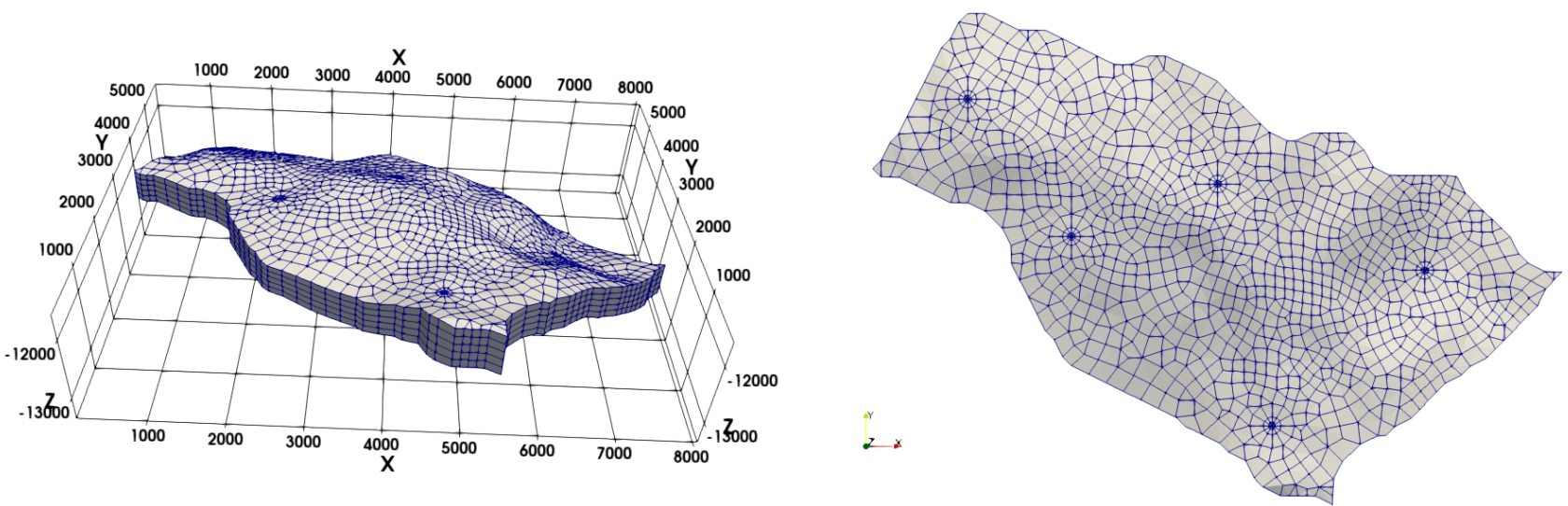

Figure 12: Synthetic reservoir: Permeability and porosity maps transferred to the fine scale mesh.
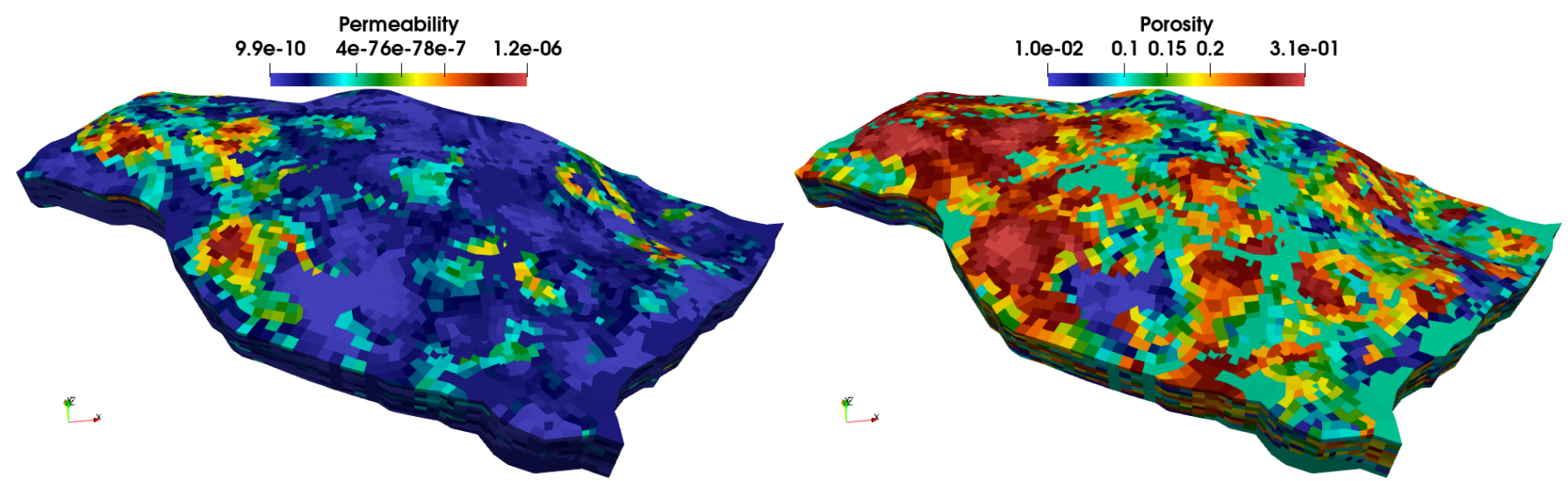

Figure 13: Synthetic reservoir: Permeability and porosity maps transferred to the fine scale mesh. 


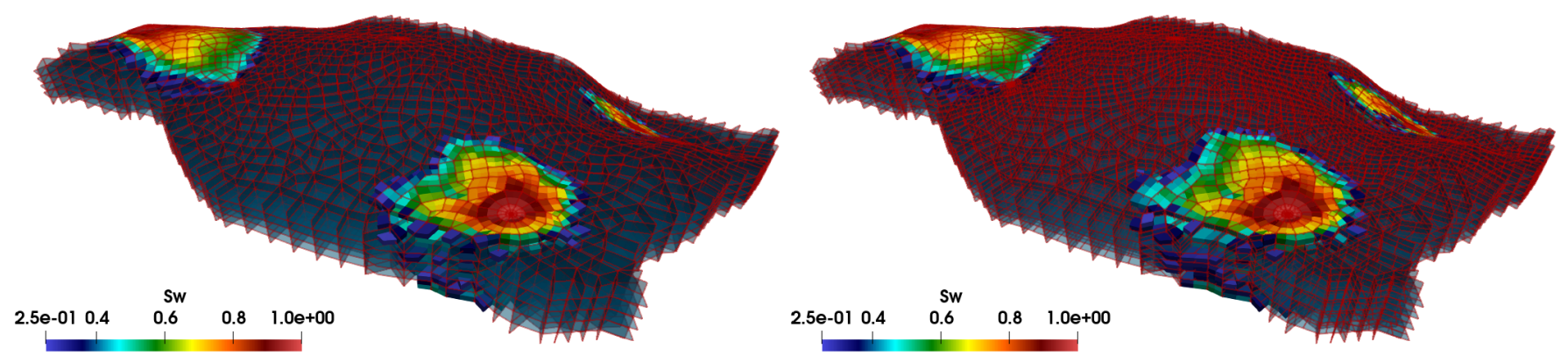

Figure 14: Synthetic reservoir: Saturation portrait at $t=9200[d]$. Used threshold $(0.25,1.0)$. Skeleton at $l=0$ (left-side) and $l=1$ right-side.
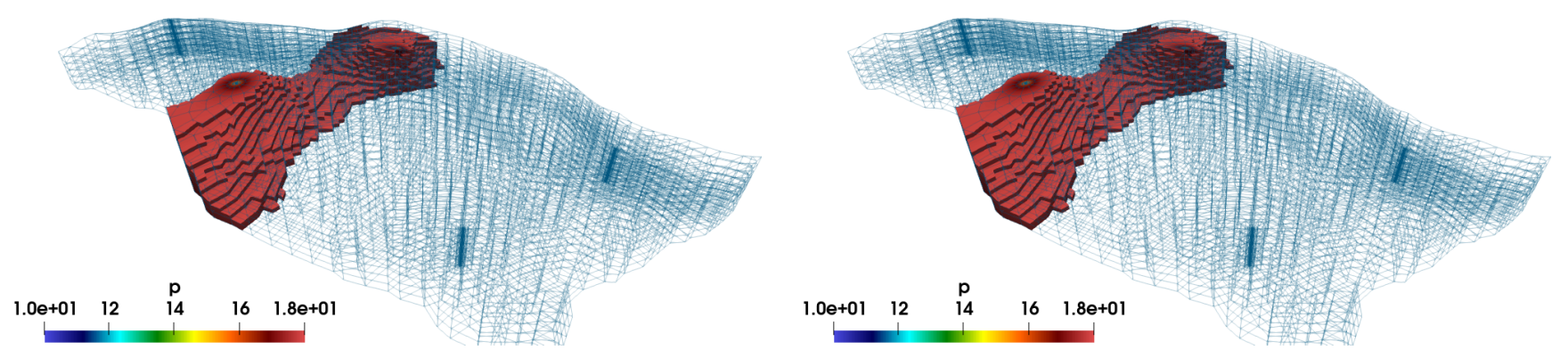

Figure 15: Synthetic reservoir: Pressure portrait at $t=9200[d]$. Used threshold $(10,18)$. Skeleton at $l=0$ (left-side) and $l=1$ right-side.

Figure 14 shows the skeleton mesh in cyan color with skeleton borders represented in red. The figure on the right side shows the skeleton adaptation for the reference solution. In addition, figure 14 shows the saturation threshold of $(0.25,1.0)$ at $t=9200[d]$. The integrated saturations over the threshold are:

- For $l=0$ is $2.8709338 \times 10^{8}$;

- For $l=1$ is $2.5085393 \times 10^{8}$.

It shows that the mass in the system is almost the same for both simulations.

Figure 15 shows the pressure threshold of $(10,18) \mathrm{MPa}$ at $t=9200[d]$. The region obtained by the threshold agrees with the reference solution.

Table 8 shows that in 3D setting the reduction for the global mixed problem is significant.

\begin{tabular}{|c|c|c|c|}
\hline Setting & DoF & DoF (SC) & $\%$ \\
\hline \hline MHM-H (div),$l=0$ & 2519804 & 88833 & 3.52 \\
\hline Reference & 2763068 & 332097 & 12.01 \\
\hline
\end{tabular}

Table 8: Synthetic reservoir: Number of equations in the condensed linear systems (DoF) for MHM-H (div) and reference mixed simulation.

\section{Concluding remarks}

This paper introduces the MHM-H(div) method for solving sequentially two-phase flow in heterogeneous porous media. The formulation results in a system of equations corresponding to the trace of the normal fluxes between macro-domains and one average pressure for each subdomain. In order to allow to use large time steps, the sequentially fully implicit algorithm SFI approximation applied to two-phase flow was adopted. For the case of two-phase flow Quasi-Newton augmented with the 
Thomas method is proposed to decrease the number of iterations for the transport module. The multiscale procedure guarantees the local conservation of mass throughout the simulation at both coarse and fine spatial scales.

Several numerical tests were presented demonstrating that pressure and saturation approximations are obtained robustly compared with those obtained from the single-scale simulations. Overall, MHM-H(div) allows to capture the spatial variation of the solution demonstrating its adequacy to represent the intrinsic spatial multiscale nature of the problem. The expressive reduction of the size of the global system of equations demonstrates that the MHM-H(div) method provides a suitable simulation technique for large-scale multiphase simulations.

Ongoing research activities explore the divide-and-conquer approach of the MHM-H(div) method for the adoption of parallel computational strategies, allowing the approximation of larger scale problems in a reasonable computational time. A posteriori error estimates for the design of adaptive multiscale structures evolving in time, extensions to more complex fluid physics characteristics (e.g. capillary effects) and discrete fracture networks are also under study.

\section{Acknowledgments}

The authors P. R. B. Devloo and S. M. Gomes gratefully acknowledge financial support from CNPq - Conselho Nacional de Desenvolvimento Científico e Tecnológico (grants 305823-2017-5, and 306167/2017-4), and from FAPESP - Fundação de Amparo à Pesquisa do Estado de São Paulo, Brazil (grant 2016/05144-0). P. R. B. Devloo and O. Durán also acknowledge financial support from ANP-Brazilian National Agency of Petroleum, Natural Gas and Biofuels (grant 2014/00090-2) and of FAPESP/Equinor (grant No 2017/15736-3). S. M. Gomes is grateful for the support and hospitality received during her visit to Universidade Federal da Paraíba, PB, Brazil, whilst this manuscript was partially prepared.

\section{References}

[1] Aziz S. Odeh. An overview of mathematical modeling of the behavior of hydrocarbon reservoirs. SIAM Review, 24(3):263-273, 1982.

[2] T. Ertekin, J.H. Abou-Kassem, and G.R. King. Basic Applied Reservoir Simulation. 2001.

[3] John R. Fanchi. Principles of Applied Reservoir Simulation. 2018.

[4] L.J. Durlofsky. Numerical calculation of equivalent grid block permeability tensors for heterogeneous porous media. Water Resources Research, 27(5):699-708, 1991.

[5] J.R. Kyte and D.W. Berry. New pseudo functions to control numerical dispersion. Soc. Pet. Eng. J., 15(04):269-276, 1975.

[6] R.E. Guzman, D. Giordano, F.J. Fayers, A. Godi, and K. Aziz. Evaluation of Dynamic Pseudo Functions for Reservoir Simulation, pages 9-19. 1996.

[7] D. Qi and T. Hesketh. An analysis of upscaling techniques for reservoir simulation. Petroleum Science and Technology, 23(7-8):827-842, 2005.

[8] T.Y. Hou and X.-H. Wu. A multiscale finite element method for elliptic problems in composite materials and porous media. Journal of Computational Physics, 134(1):169-189, 1997.

[9] Z. Chen and T. Y. Hou. A mixed multiscale finite element method for elliptic problems with oscillating coefficients. Math. Comput., 72(242):541-576, apr 2003.

[10] P. Jenny, S.H. Lee, and H.A. Tchelepi. Multi-scale finite-volume method for elliptic problems in subsurface flow simulation. Journal of Computational Physics, 187(1):47-67, 2003. 
[11] V. A. Badri Narayanan and N. Zabaras. Variational multiscale stabilized fem formulations for transport equations: Stochastic advection-diffusion and incompressible stochastic navier-stokes equations. J. Comput. Phys., 202(1):94-133, January 2005.

[12] J. E. Aarnes, S. Krogstad, and K.-A. Lie. A hierarchical multiscale method for two-phase flow based upon mixed finite elements and nonuniform coarse grids. Multiscale Modeling $\&$ Simulation, 5(2):337-363, 2006.

[13] T. Arbogast and K. J. Boyd. Subgrid upscaling and mixed multiscale finite elements. SIAM Journal on Numerical Analysis, 44(3):1150-1171, 2006.

[14] J. E. Aarnes, V. Kippe, K.-A. Lie, and A. B. Rustad. Modelling of Multiscale Structures in Flow Simulations for Petroleum Reservoirs.

[15] J. E. Aarnes and Y. Efendiev. A multiscale method for modeling transport in porous media on unstructured corner-point grids. J. Algorithms Comput. Technol., 2(2):299-318, 2008.

[16] Y. Efendiev and T. Y. Hou. Multiscale Finite Element Methods: Theory and Applications: 4 (Surveys and Tutorials in the Applied Mathematical Sciences). 2009.

[17] L. Delpopolo Carciopolo, L. Formaggia, A. Scotti, and H. Hajibeygi. Conservative multirate multiscale simulation of multiphase flow in heterogeneous porous media. Journal of Computational Physics, 404:109134, 2020.

[18] O. Duran, P. R. B. Devloo, S. M. Gomes, and F. Valentin. A multiscale hybrid method for darcy's problems using mixed finite element local solvers. Computer Methods in Applied Mechanics and Engineering, 354:213-244, 2019.

[19] G. Chavent and G. Salzano. A finite-element method for the 1-d water flooding problem with gravity. Journal of Computational Physics, 45(3):307-344, 1982.

[20] L. J. Durlofsky. A triangle based mixed finite element-finite volume technique for modeling two phase flow through porous media. Journal of Computational Physics, 105(2):252-266, 1993.

[21] T. Arbogast, L. C. Cowsar, M. F. Wheeler, and I. Yotov. Mixed finite element methods on nonmatching multiblock grids. SIAM Journal on Numerical Analysis, 37(4):1295-1315, 2000.

[22] T. Arbogast and S. L. Bryan. A two-scale numerical subgrid technique for water flood simulations. SPE J., 7:446-457, 2002.

[23] M. F. Wheeler and M. Peszyńska. Computational engineering and science methodologies for modeling and simulation of subsurface applications. Advances in Water Resources, 25(8):1147$1173,2002$.

[24] S. Sun, B. Rivière, and M. F. Wheeler. A Combined Mixed Finite Element and Discontinuous Galerkin Method for Miscible Displacement Problem in Porous Media, pages 323-351. Springer US, Boston, MA, 2002.

[25] C. Dawson, S. Sun, and M. F. Wheeler. Compatible algorithms for coupled flow and transport. Computer Methods in Applied Mechanics and Engineering, 193(23):2565-2580, 2004.

[26] T. Arbogast, G. Pencheva, M. F. Wheeler, and I. Yotov. A multiscale mortar mixed finite element method. Multiscale Modeling \&S Simulation, 6(1):319-346, 2007.

[27] S. F. Matringe, R Juanes, and H. A. Tchelepi. Mixed Finite Element and Related Control-Volume Discretizations for Reservoir Simulation on Three-Dimensional Unstructured Grids, pages 1-13. 2007. 
[28] M. F. Wheeler, G. Xue, and I. Yotov. A multiscale mortar multipoint flux mixed finite element method. ESAIM: M2AN, 46(4):759-796, 2012.

[29] A. S. Abushaikha, D. V. Voskov, and H. A. Tchelepi. Fully implicit mixed-hybrid finite-element discretization for general purpose subsurface reservoir simulation. Journal of Computational Physics, 346:514-538, 2017.

[30] M. R. Correa and M. A. Murad. A new sequential method for three-phase immiscible flow in poroelastic media. Journal of Computational Physics, 373:493-532, 2018.

[31] G. Singh, W. Leung, and M. F. Wheeler. Multiscale methods for model order reduction of non-linear multiphase flow problems. Computational Geosciences, 23:305-323, 2019.

[32] O. Duran, M. Sanei, P. R. B. Devloo, and E. S. R. Santos. An enhanced sequential fully implicit scheme for reservoir geomechanics. Computational Geosciences, 2020.

[33] C. Harder, D. Paredes, and F. Valentin. A family of multiscale hybrid-mixed finite element methods for the darcy equation with rough coefficients. Journal of Computational Physics, 245:107-130, 2013.

[34] P. Devloo, W. Teng, and C. Zhang. Multiscale hybrid-mixed finite element method for flow simulation in fractured porous media. CMES Comput. Model. Eng. Sci., 1:145-163, 2019.

[35] O. Duran. Development of a surrogate multiscale reservoir simulator coupled with geomechanics, 2017.

[36] P. Jenny, S. H. Lee, and H. A. Tchelepi. Adaptive multiscale finite-volume method for multiphase flow and transport in porous media. Multiscale Modeling \& Simulation, 3(1):50-64, 2005.

[37] A. Moncorgé, H.A. Tchelepi, and P. Jenny. Sequential fully implicit formulation for compositional simulation using natural variables. Journal of Computational Physics, 371:690-711, 2018 .

[38] J. Jiang and H. A. Tchelepi. Nonlinear acceleration of sequential fully implicit (sfi) method for coupled flow and transport in porous media. Computer Methods in Applied Mechanics and Engineering, 352:246-275, 2019.

[39] J. Kim, H. A. Tchelepi, and R. Juanes. Stability and convergence of sequential methods for coupled flow and geomechanics: Drained and undrained splits. Computer Methods in Applied Mechanics and Engineering, 200(23):2094-2116, 2011.

[40] J. Wan, L. J. Durlofsky, T. J. R. Hughes, and K. Aziz. Stabilized Finite Element Methods for Coupled Geomechanics-Reservoir Flow Simulations, pages 1-11. 2003.

[41] J. A. White and R. I. Borja. Stabilized low-order finite elements for coupled soliddeformation/fluid-diffusion and their application to fault zone transients. Computer Methods in Applied Mechanics and Engineering, 197(49):4353-4366, 2008.

[42] J. Choo and R. I. Borja. Stabilized mixed finite elements for deformable porous media with double porosity. Computer Methods in Applied Mechanics and Engineering, 293:131-154, 2015.

[43] Z. Chen. Formulations and numerical methods of the black oil model in porous media. SIAM J. Numer. Anal., 38(2):489-514, 2000.

[44] Y. Brenier and J. Jérôme. Upstream differencing for multiphase flow in reservoir simulation. SIAM Journal on Numerical Analysis, 28(3):685-696, 1991. 
[45] S. H. Lee, Y. Efendiev, and H. A. Tchelepi. Hybrid upwind discretization of nonlinear two-phase flow with gravity. Advances in Water Resources, 82:27-38, 2015.

[46] J. Degroote, R. Haelterman, S. Annerel, P. Bruggeman, and J. Vierendeels. Performance of partitioned procedures in fluid-structure interaction. Computers $\mathscr{E}$ Structures, 88(7):446-457, 2010 .

[47] A. E. J. Bogaers, S. Kok, B. D. Reddy, and T. Franz. An evaluation of quasi-newton methods for application to fsi problems involving free surface flow and solid body contact. Computers 8 Structures, 173:71-83, 2016.

[48] T. Liu, S. Bouaziz, and L. Kavan. Quasi-newton methods for real-time simulation of hyperelastic materials. ACM Trans. Graph., 36(3):1-16, 2017.

[49] S. W. Sloan, D. Sheng, and A. J. Abbo. Accelerated initial stiffness schemes for elastoplasticity. International Journal for Numerical and Analytical Methods in Geomechanics, 24(6):579-599, 2000 .

[50] A. Cordero, J. L. Hueso, E. Martínez, and J. R. Torregrosa. Increasing the convergence order of an iterative method for nonlinear systems. Applied Mathematics Letters, 25(12):2369-2374, 2012 .

[51] X.-Y. Xiao and H.-W. Yin. Accelerating the convergence speed of iterative methods for solving nonlinear systems. Applied Mathematics and Computation, 333:8-19, 2018.

[52] M. Vohralík and B. I. Wohlmuth. Mixed finite element methods: Implementation with one unknown per element, local flux expressions, positivity, polygonal meshes, and relations to other methods. Mathematical Models and Methods in Applied Sciences, 23(05):803-838, 2013.

[53] M. Khoshniat, G. R. Stuhne, and D. A. Steinman. Relative Performance of Geometric Search Algorithms for Interpolating Unstructured Mesh Data. 2003.

[54] A. M. Farias, P. R. B. Devloo, S. M. Gomes, and O. Duran. An object-oriented framework for multiphysics problems combining different approximation spaces. Finite Elements in Analysis and Design, 151:34-49, 2018.

[55] M. Hayek, E. Mouche, and C. Mügler. Modeling vertical stratification of co2 injected into a deep layered aquifer. Advances in Water Resources, 32(3):450-462, 2009.

[56] J. W. Both, K. Kumar, J. M. Nordbotten, and F. A. Radu. Anderson accelerated fixed-stress splitting schemes for consolidation of unsaturated porous media. Computers $\&$ Mathematics with Applications, 77(6):1479-1502, 2019. 7th International Conference on Advanced Computational Methods in Engineering (ACOMEN 2017).

[57] G. D. Avansi and D. Schiozer. Unisim-i: Synthetic model for reservoir development and management applications. Int. J. Model. Simul. Pet. Ind., 9:21-30, 2015. 